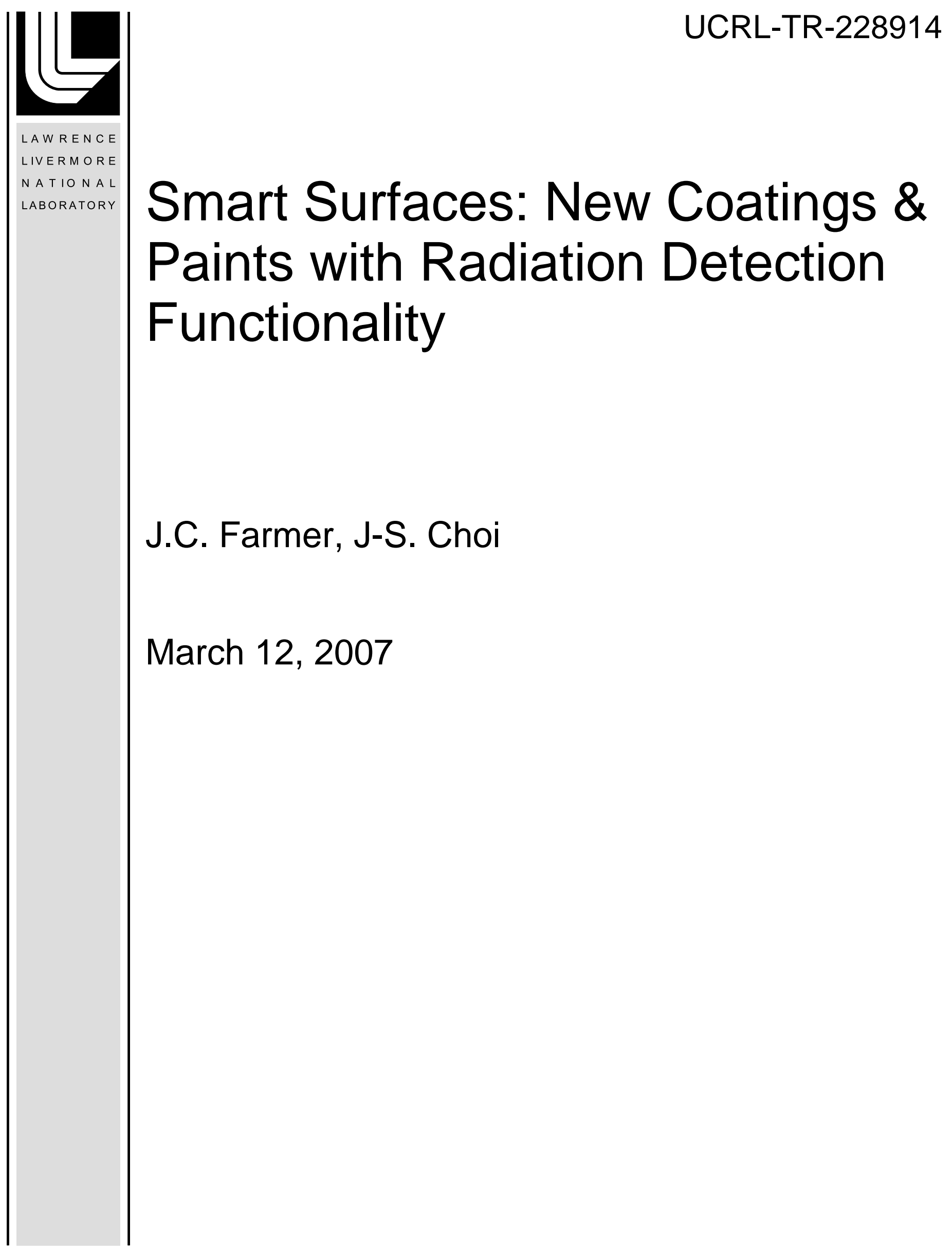


This document was prepared as an account of work sponsored by an agency of the United States Government. Neither the United States Government nor the University of California nor any of their employees, makes any warranty, express or implied, or assumes any legal liability or responsibility for the accuracy, completeness, or usefulness of any information, apparatus, product, or process disclosed, or represents that its use would not infringe privately owned rights. Reference herein to any specific commercial product, process, or service by trade name, trademark, manufacturer, or otherwise, does not necessarily constitute or imply its endorsement, recommendation, or favoring by the United States Government or the University of California. The views and opinions of authors expressed herein do not necessarily state or reflect those of the United States Government or the University of California, and shall not be used for advertising or product endorsement purposes.

This work was performed under the auspices of the U.S. Department of Energy by University of California, Lawrence Livermore National Laboratory under Contract W-7405-Eng-48. 


\title{
SMART SURFACES: NEW COATINGS \& PAINTS WITH RADIATION DETECTION FUNCTIONALITY
}

\author{
Joseph Farmer and Jor-Shan Choi \\ Nuclear Science \& Systems Engineering Program \\ Energy and Environment Directorate \\ Lawrence Livermore National Laboratory \\ Livermore, California 94550
}

\begin{abstract}
Paints are being developed and tested that might ultimately be able to detect radiological agents in the environment by incorporating special pigments into an organic polymeric binder that can be applied as a paint or coatings. These paints detect radioactive sources and contaminants with inorganic or organic scintillation or thermo-luminescent pigments, which are selected based upon the radiation $(\alpha, \beta, \gamma$ or $n)$ to be detected, and are shown in Figure 1.
\end{abstract}
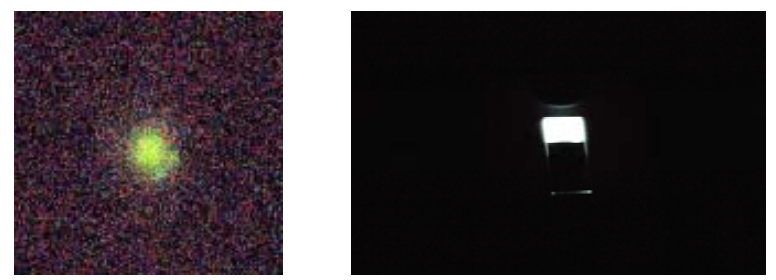

Figure 1 - Left image shows radiation source positioned behind a panel painted with scintillationtype paint. Right image shows luminescence due to historic dose experienced by thermo-luminescent-type paint.

\section{INTRODUCTION}

Since the surfaces of most engineered structures are painted to protect them from corrosion, and to impart esthetic beauty, paints provide a viable means for imparting such functionality to surfaces. Ultimately, such paints could be used to allow relatively easy detection of widespread radioactive contamination on the surfaces of buildings and mass transit systems, thereby enabling such areas to be more readily decontaminated. Such coatings could also be used to form large-area temper-proof indicators, to reveal the presence of radioactive materials, and to ensure compliance with various regulatory requirements and other agreements. Such paints would be ideal for buildings and facilities involved in the processing and storage of nuclear materials, and could even be used to monitor contamination and dose on-board space craft and inside nuclear powered ships. Developmental work done thus far has demonstrated the viability of this concept. The concept of using sensing pigments can be easily extended to molded plastic parts. Additional work required for the full realization of the concepts potential is outlined.

The first demonstration of a radiation-sensitive paint involved the successful detection of scintillations from painted surfaces irradiated by both alpha particles and gamma rays. Alpha particles from a weak 1-nCi Pu-239 source were detected with a scintillation-type paint, a photomultiplier tube (PMT), and a pulse counting network. Gamma rays from a $100-\mu \mathrm{Ci}$ radium-226 source were also detected with different scintillation-type paint by performing timelapse photography with a commercially available 12.8-megapixel camera.

More recently, radiation sensitive paints capable of recording dose have been developed. These paints have been prepared with the three size-fraction thermo-luminescent pigments: coarse $(-75 /+53 \mu \mathrm{m})$; medium $(-53 /+38 \mu \mathrm{m})$; and fine $(-38 \mu \mathrm{m})$. As previously discussed, each coating was prepared with powder that was produced by milling TLD-700 Li-7 fluoride $\left({ }^{7} \mathrm{LiF}\right)$ chips, taking the desired size fraction, and mixing it with a clear organic paint for hightemperature applications (PJH Brands SP-115 VHT Clear Coat). In all cases, the ratio of clear coat to powder was 10:4 by weight.

First, the coating pigment was given a dose of $>20,000$ rad from a Co-60 gamma source, mixed with the clear coat paint, and then applied to a surface. The surface was then heated with a hotplate while the thermo-luminescent response was monitored with a photomultiplier tube. The temperature thresholds of these $\gamma$-sensitive pains were determined to be between 110 to $120^{\circ} \mathrm{C}$. The signal intensities obtained with coarse $(-75 /+53 \mu \mathrm{m})$ and medium $(-53 /+38 \mu \mathrm{m})$ pigment were greater than that obtained with the fine $(<38 \mu \mathrm{m})$ pigment. A slight optimum in performance may exist for the $-53 /+38 \mu \mathrm{m}$ pigment, with the fine pigment having inferior performance.

Each sample was then irradiated a second time with a dose of $\sim 50,000 \mathrm{rad}$ and interrogated. In this case, the temperature threshold for thermoluminescence appeared to be slightly lower ( $\sim 80$ to $100^{\circ} \mathrm{C}$ ) than observed during the initial irradiations and interrogations. However, additional work is required to be certain. Here too the signal intensity from the fine $(<38 \mu \mathrm{m})$ pigment was less than that obtained with the coarser $(-75 /+53$ and $-53 /+38 \mu \mathrm{m})$ 
pigments, which is consistent with the initial irradiation. Once again, a slight optimum in performance existed for the $-53 /+38 \mu \mathrm{m}$ size pigment, with the fine pigment having inferior performance.

A third irradiation of the thermo-luminescent paints delivered 5000-rad dose. In this case, the observed temperature threshold for thermoluminescence was approximately 120 to $150^{\circ} \mathrm{C}$, and was greater than the threshold of approximately 80 to $100{ }^{\circ} \mathrm{C}$ observed with the 50,000-rad dose. It was therefore concluded that the stored dose information is more stable as the dose decreases.

It was also concluded that the performance of pigments in $\mathrm{LiF}(\# 1)$ and $\mathrm{LiF}(\# 2)$ were comparable, whereas the performance of pigment in $\mathrm{LiF}$ (\#3) was relatively poor. The size distribution of pigments used in $\operatorname{LiF}(\# 1)$ and $\operatorname{LiF}(\# 2)$ were coarse $(-75 /+53 \mu \mathrm{m})$ and medium $(-53 /+38 \mu \mathrm{m})$, respectively. The size distribution of the pigment used in $\mathrm{LiF}$ (\#2) was fine $(-38 \mu \mathrm{m})$. It was therefore concluded that radiation sensitive paints using this type of thermo-luminescent pigment should use particle sizes greater than $38 \mu \mathrm{m}$. The finer pigments may have had too many defects from grinding to function well as thermo-luminescent micro crystals. The peak emission during thermoluminescence occurred at a wavelength of $400 \mathrm{~nm}$, which is in the violet-to-ultraviolet range.

\section{NOMENCLATURE}

Charge-Coupled Device

Photomultiplier Tube

Thermo-Luminescence

CCD

Thermo-Luminescence Detector

PMT

Ultraviolet

TL

TLD

Visible

UV

VIS

\section{TECHNICAL APPROACH}

Protective paints and coatings have been developed that could ultimately be used to detect the presence of radiation on surfaces, or in close proximity to surfaces. Scintillation-type paints enable the instantaneous detection of radiological agents in the environment, present on or near surfaces through scintillation. Special inorganic pigments are added to paints that produce luminescence when irradiated by alpha, beta and gamma rays. Organic scintillation compounds can also be blended into the organic binders. Signal enhancement and wavelength shifting can be accomplished by using the luminescence to stimulate florescence in dyes incorporated into the polymeric paint or binder. Integrating paints and coatings are being developed to enable extremely sensitive dose recording due to any historic exposure to radiation. The second type of paint, referred to here as thermo-luminescent, records accumulated dose. In this case, an inorganic thermo-luminescent pigment produces light in the visible-ultraviolet spectrum that is proportional to radiation exposure, and can be reset by annealing. High temperature transparent (clearcoat) paints serve as binder and host matrix for scintillation and thermo-luminescent pigments, and should not absorb the emitted luminescence.

The presence of radiological agents on a surface can be detected through the incorporation of scintillation or thermo-luminescent materials listed below into a clear organic paint, thereby imparting radiation sensitivity, as shown in Figure 2. Special inorganic pigments are added to the paint that produce luminescence when irradiated by alpha and gamma rays [1-5], while organic scintillation pigments are exploited for the detection of beta particles [1]. To enhance detection, the luminescence can be used to stimulate florescence in dyes within the polymeric binder of the paint, thereby shifting the wavelength of emitted light.

Inorganic Scintillator Pigments

- $\quad \mathrm{LiI}(\mathrm{Sn})-\mathrm{n}$ detection

- $\mathrm{ZnS}(\mathrm{Ag})-\alpha$ detection

- $\mathrm{NaI}(\mathrm{Tl})-\gamma$ detection

- $\quad \mathrm{CsI}(\mathrm{Tl}), \mathrm{CsI}(\mathrm{Na})-\gamma$ detection

- $\mathrm{CaF}_{2}(\mathrm{Eu})-\gamma$ detection

- $\mathrm{BGO}-\gamma$ detection

- $\mathrm{BaF}_{2}-\gamma$ detection

Organic Scintillator Pigments

- $\quad$ anthracene $-\beta, \mathrm{n}$ detection

- trans-stilibene $-\beta$ detection

- $\quad$-terphenyl $-\beta$ detection

- diphenylorazole $-\beta, \mathrm{n}$ detection

- $\quad$ tetraphenyl butadiene $-\beta$ detection

- terphenyl in polystyrene $-\beta$ detection

Any historical exposure of a surface to radiation can be detected through the incorporation of a thermo-luminescent pigment into a transparent paint. These integrating coatings record the historic dose that the paint has seen prior to the thermal annealing that is used to reset the material. Note that more recent advancements in thermo-luminescent materials have lead to the possibility of using aluminum oxide with interrogation and reset accomplished with lightstimulated luminescence [6].

Thermo-Luminescent Pigments

- $\mathrm{CaSO}_{4}(\mathrm{Tm})$ - thermo- luminescence

- $\quad{ }^{7} \mathrm{Li}_{2} \mathrm{~B}_{4} \mathrm{O}_{7}(\mathrm{Cu})$ - thermo-luminescence

- ${ }^{6} \mathrm{Li}_{2} \mathrm{~B}_{4} \mathrm{O}_{7}(\mathrm{Cu})$ - thermo- luminescence

- $\quad{ }^{7} \mathrm{LiF}(\mathrm{Tl} / \mathrm{Mg})$ - thermo-luminescence

- $\quad{ }^{7} \mathrm{LiF}(\mathrm{Cu})$ - thermo-luminescence

- $\mathrm{Al}_{2} \mathrm{O}_{3}$ - photon-induced luminescence 
Thermo-luminescent (TL) paints have been prepared with the three size-fraction TL pigments: coarse $(-75 /+53 \mu \mathrm{m})$; medium $(-53 /+38 \mu \mathrm{m})$; and fine $(-38 \mu \mathrm{m})$. Each coating was prepared with powder that was produced by milling TLD-700 Li-7 fluoride $\left({ }^{7} \mathrm{LiF}\right)$ chips, taking the desired size fraction, and mixing it with a clear organic paint for hightemperature applications (PJH Brands SP-115 VHT Clear Coat). In all cases, the ratio of clear coat to powder was 10:4 by weight.

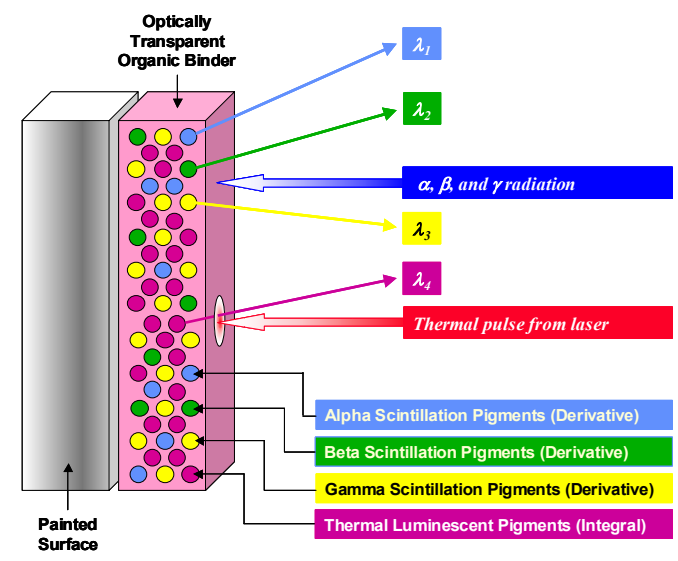

Figure 2 - This figure is a schematic representation of a "Smart Surface" or "Intellicoat" showing organic-based paint with various scintillation pigments that provide instantaneous response to the presence of radiation (derivative-type coating). A fourth thermo-luminescent (also referred to as thermal luminescent) pigment scintillates during heating, thereby releasing a quantity of light proportional to dose. In this case, heating is used to first release the "recorded" integrated signal that is proportional to the total radiation exposure over an extended period of time. This progress report describes recent work on thermo-luminescent paints, which promise substantial sensitivity through measurement of integrated dose, as well as early results with scintillation paints. These concepts and approaches are considered proprietary information of Lawrence Livermore National Laboratory (see auspices).

Scintillation-Type Paints - The selection of an ideal scintillation pigment for a radiation-sensitive paint is difficult. A material must be found that simultaneously satisfies several important criteria: high photon yield from the radiation being detected; a peak emission wavelength that is well suited to the spectral sensitivity of the interrogation device; a refractive index at the peak wavelength that closely matches the organic binder, thus minimizing losses due to scattering; and exceptional long-term stability in the presence of water. In regard to stability in the presence of water, hygroscopic materials are obviously incompatible with water-based latex clear coats, and lack the long-term stability necessary for enduring humid and moist conditions. In cases where these scintillation materials need to be used, it is necessary to disperse them in lacquers that are dissolved in organic solvents. Refractive index matching between the binder and the pigment particles would minimize the effects of light scattering at interfaces, and would therefore maximize the transmission of emitted light towards the detector. Properties for several of the scintillation materials listed on the previous page are given in Table 1.

Of all known scintillation materials, thallium-doped sodium iodide, $\mathrm{NaI}(\mathrm{Tl})$, is best matched to the spectral sensitivity of photomultiplier tubes (PMTs) with a bialkali photocathodes $[3,7]$. Since the wavelength of maximum emission for $\mathrm{NaI}(\mathrm{Tl})$ is close to the peak spectral sensitivity such photo-multipliers, it has been used to build the most sensitive PMT-based detectors. Unfortunately, this highly responsive scintillation material is very hygroscopic, and is therefore undesirable as a scintillation pigment in the new paints discussed here, especially in water-based latex paints. Since these crystals can be damaged by intense solar radiation, their application in paints is further complicated.

In comparison to $\mathrm{NaI}(\mathrm{Tl})$, thallium-doped cesium iodide, $\mathrm{CsI}(\mathrm{Tl})$ is significantly less hygroscopic, and might therefore be useful as a scintillation pigment. Unfortunately, CsI(Tl) scintillation crystals with PMT detectors produce only $45 \%$ of the output possible with comparable $\mathrm{NaI}(\mathrm{Tl})$-based systems [4,7]. While $\mathrm{CsI}(\mathrm{Tl})$ is one of the brightest known scintillation materials, with a light yield of approximately 54 photons per $\mathrm{keV}$, compared to only 38 photons per $\mathrm{keV}$ for $\mathrm{NaI}(\mathrm{Tl})$, its wavelength of maximum emission is centered at $550 \mathrm{~nm}$. $\mathrm{CsI}(\mathrm{Tl})$ is therefore poorly matched to the spectral response of a typical PMT with a bialkali photocathode. This relatively long-wavelength scintillation material is however very well suited to the spectral sensitivity of solidstate photodiodes. While the relatively slow decay time for $\mathrm{CsI}(\mathrm{Tl})$ limits its application in high count rate applications, it is not viewed as being problematic in the application at hand. It is important to note that the use of sodium instead of thallium as an impurity for doping CsI leads to unacceptable sensitivity to moisture.

The relatively high stability of europium-doped calcium fluoride, $\mathrm{CaF}_{2}(\mathrm{Eu})$, in the presence of water makes it highly desirable as a potential scintillation pigment in a paint $[5,7]$. However, the disadvantage of this particular pigment is the relatively low light yield (19 photons per $\mathrm{keV}$ ). The light output for a PMT with a bialkali photocathode is comparable to that from a $\mathrm{CsI}(\mathrm{Tl})$ based system. These will be incorporated into the paint films, thereby imparting 
radiation sensitivity. Other active agents will also be exploited.

Thermo-Luminescent Paint - The standard 802 dosimeter used at LLNL and elsewhere is based upon three TL-type materials: $\mathrm{CaSO}_{4}(\mathrm{Tm}) ;{ }^{7} \mathrm{Li}_{2} \mathrm{~B}_{4} \mathrm{O}_{7}(\mathrm{Cu})$; and ${ }^{6} \mathrm{Li}_{2} \mathrm{~B}_{4} \mathrm{O}_{7}(\mathrm{Cu})$, which are hygroscopic [6]. The calcium-based TL material is 30 -times more sensitive than the lithium-based material. Older TL dosimeters used lithium fluoride, dopoed with either tellurium, magnesium, or copper: ${ }^{7} \mathrm{LiF}(\mathrm{Tl} / \mathrm{Mg})$; and ${ }^{7} \mathrm{LiF}(\mathrm{Cu})$. New TL dosimeters use aluminum oxide with lightinduced photoemission: $\mathrm{Al}_{2} \mathrm{O}_{3}$. Plastic resins can be used for neutron dosimeters. For example, "Columbia Grade Resin 39 (CR 39)" plastic resin is used to image neutron tracks due to heavy charge particles produced by $n-p$ reactions. Tracks are imaged (blown up) with reagents during reaction in oven.

Applying Paints - Numerous methodologies can be used for the production of derivative-type paints and coatings for detecting the presence of radiological agents on or near surfaces, and for the production of "integrating" paints and coatings for quantifying longterm exposure to doses of radiation, which may or may not be present at the time of interrogation [8]. These coatings incorporate scintillation and/or thermo-luminescent materials as pigments and can be easily produced with a variety of processes summarized below:

Organic polymeric binders

- Spray-on paints or coatings with organic polymeric binders

- Brush-on paints or coatings with organic polymeric binders

- Coatings and films produced with web coater and organic polymeric binders

- Powder coatings

Inorganic ceramic/metallic binders

- Cold-spray processes

- Thermal-spray processes

Shingleton and LaFrate [6] have recommended the use of standard clear decontamination paints that can be easily pealed from surface as single sheet for retrieval and reading. These paints are used in the United Kingdom for preventing contamination of systems in nuclear service, and for aiding in enabling easy decontamination during after work is complete.
Detection - The paints and coatings can be interrogated by any one of several means. These include, but are not limited to:

Paints with Scintillation Pigments

- Photomultiplier Tube (PMT) with Amplifier \& Pulse Counting

- CCD Array Camera

Paints with Thermo-Luminescent Pigments

- Laser Pulse Heating or Activation with CCD Array Camera Sensor (or PMT)

- Microscopic Heating Filaments with CCD Array Camera (or PMT)

Scintillation provides an opportunity to perform spectroscopy, since the height of the output pulse from the PMT amplifier is proportional to the energy of the emitted radiation. This energy-dependent pulse height can be fed to upper and lower discriminators, with anti-coincidence counting. Such a scheme only counts those pulses falling between two energy limits determined by the settings on the discriminators. 
Table 1-Published Properties of Scintillation Material [1-5]

\begin{tabular}{|c|c|c|c|c|c|c|c|c|c|c|c|}
\hline Material & Detected & $\rho$ & $\begin{array}{c}\text { Melting } \\
\text { Point }\end{array}$ & $\begin{array}{c}\lambda \\
\text { Maximum } \\
\text { Emission }\end{array}$ & Cutoff & $\begin{array}{l}\text { Refractive } \\
\text { Index }\end{array}$ & $\begin{array}{l}\text { Decay } \\
\text { Time }\end{array}$ & Light Yield & $\begin{array}{c}\text { Light } \\
\text { Output }\end{array}$ & $\begin{array}{l}\text { Hygro- } \\
\text { scopic }\end{array}$ & Hardness \\
\hline & & $\mathrm{g} / \mathrm{cm}^{3}$ & Kelvins & $\mathrm{Nm}$ & $\mathrm{nm}$ & None & $\mu \mathrm{sec}$ & $\begin{array}{c}\text { Photons per } \\
\text { keV }\end{array}$ & $\begin{array}{l}\text { Percent } \\
\text { NaI(Tl) }\end{array}$ & & Mho \\
\hline $\mathrm{NaI}(\mathrm{Tl})$ & $\gamma$ & 3.67 & 924 & 415 & & 1.85 & 0.25 & 38 & 100 & Yes & 2 \\
\hline CsI(Tl) & $\gamma$ & 4.51 & 894 & 550 & 320 & 1.79 & 1.00 & 54 & 45 & Slight & 2 \\
\hline $\mathrm{CsI}(\mathrm{Na})$ & $\gamma$ & 4.51 & 894 & 420 & 300 & 1.84 & 0.63 & 41 & 85 & Yes & 2 \\
\hline CsI & $\gamma$ & 4.51 & 894 & 315 & 260 & 1.95 & 0.016 & 2 & $4-6$ & Slight & 2 \\
\hline $\mathrm{CaF}_{2}(\mathrm{Eu})$ & $\gamma$ & 3.18 & 1691 & 435 & 395 & 1.47 & 0.94 & 19 & 50 & No & 4 \\
\hline $\mathrm{ZnS}(\mathrm{Ag})$ & $\alpha$ & 4.09 & 1700 & 450 & & 2.36 & 0.110 & 50 & 130 & No & Unknown \\
\hline
\end{tabular}

Table 2 - Published Values for Radiation Sources [9-14]

\begin{tabular}{|c|c|c|c|c|c|c|}
\hline Source & $\begin{array}{c}\text { Decay } \\
\text { Product }\end{array}$ & $\begin{array}{c}\text { Decay } \\
\text { Mode }\end{array}$ & $\begin{array}{c}\text { Decay } \\
\text { Energy }\end{array}$ & Half Life & $\begin{array}{c}\text { Specific } \\
\text { Activity }\end{array}$ & Characteristic Radiation \& Energies \\
\hline & & & $\mathrm{MeV}$ & $\mathrm{t}_{1 / 2}$ (years) & SpA (Ci/g) & $\alpha, \beta, \gamma(\mathrm{MeV})$ \\
\hline${ }_{94} \mathrm{Pu}^{239}$ & ${ }_{92} \mathrm{U}^{235}$ & $\alpha$ & 5.243 & 24,113 & 0.06204 & $\alpha(5.107), \alpha(5.145), \alpha(5.157)$ \\
\hline${ }_{17} \mathrm{Cl}^{36}$ & ${ }_{18} \mathrm{Ar}^{36}$ & $\beta$ & 0.714 & 301,200 & 0.03299 & $\beta(0.2788)$ \\
\hline${ }_{27} \mathrm{Co}^{60}$ & ${ }_{28} \mathrm{Ni}^{60}$ & $\beta, \gamma$ & 2.819 & 5.27 & & $\beta(1.173226), \gamma(1.332483)$ \\
\hline${ }_{55} \mathrm{Cs}^{137}$ & ${ }_{56} \mathrm{Ba}^{137}$ & $\beta, \gamma$ & 1.176 & 30.23 & 87.0 & $\alpha(4.601), \alpha(4.784), \gamma(0.186)$ \\
\hline${ }_{88} \mathrm{Ra}^{226}$ & ${ }_{88} \mathrm{Ra}^{226}$ & $\alpha, \gamma$ & & 1,602 & $1.39 \times 10^{8}$ & $\alpha(5.49)$ \\
& ${ }_{86} \mathrm{R}^{222}$ & $\alpha$ & & 3.823 days & & $\alpha(6.00)$ \\
& ${ }_{84} \mathrm{Po}^{218}$ & $\alpha$ & & 26.05 minutes & & $\beta(0.65), \beta(0.71), \beta(0.98), \gamma(0.295), \gamma(0.352)$ \\
& ${ }_{82} \mathrm{~Pb}^{214}$ & $\beta, \gamma$ & & 19.7 minutes & & $\beta(1.0), \beta(1.51), \beta(3.26), \gamma(0.609), \gamma(1.120), \gamma(1.764)$ \\
& ${ }_{83} \mathrm{Bi}^{214}$ & $\beta, \gamma$ & & 1.3 minutes & & $\beta(1.3), \beta(1.9), \beta(2.3), \gamma(0.296), \gamma(0.795), \gamma(1.31)$ \\
& ${ }_{81} \mathrm{Tl}^{210}$ & $\beta, \gamma$ & & &
\end{tabular}




\section{SCINTILLATION-TYPE PAINT}

Radiation Sources Used - Several radiation sources were used to evaluate the radiation-sensitive paints. The invariant properties of these sources are summarized in Table 2. In regard to Table 2, it should be noted that sealed sources such as Ra-226 transmit only gamma rays to the detector. The specific identification of sources and their activities given here:

Pu-239 Alpha Source

- Serial Number: 600/88

- Date of Preparation: September 2, 1988

- Activity: $\sim 1 \mathrm{nCi}(2810$ counts per minute)

Cl-36 Beta Source

- Serial Number: Unknown

- Date of Preparation: September 1966

- Activity: $\sim 100 \mathrm{nCi}(0.085 \mu \mathrm{Ci})$

Cs-137 Gamma Source

- Serial Number: GL732

- Date of Preparation: Unknown

- Activity: $\sim 10 \mu \mathrm{Ci}$

Ra-226 Gamma Source

- Serial Number: Unknown

- Date of Preparation: Unknown

- Activity: $\sim 100 \mu \mathrm{Ci}$

Alpha Sensitivity - The alpha-sensitive coating was made by mixing silver-doped zinc sulfide, $\mathrm{ZnS}(\mathrm{Ag})$, with a water-based polyurethane clear-coat paint . The $\mathrm{ZnS}(\mathrm{Ag})$ powder was obtained from Saint Gobain Crystals and Detectors of Newbury, Ohio, Part Number SP-1108, and was identified as Type Number Z151, received at LLNL on July $7^{\text {th }} 2005$. The water-based clear-coat interior paint that was used as a binder for the zinc sulfide pigment is tradenamed "Diamond Elite Water-Based (WB) Interior" and was selected since it had no ultraviolet inhibitors (absorbers). This paint was obtained from RustOelum Corporation of Vernon Hills, Illinois, as was marked with identification number 200041 dated November 2003. Based upon the Material Safety Data Sheet (MSDS), it is believed that this paint contains water, dipropylene glycol monomethyl ether, and dipropylene glycol monobutyl ether [15].

The ratio of $\mathrm{ZnS}(\mathrm{Ag})$ to the polyurethane clearcoat was approximately $3: 1$. For example, about $1 / 2$ teaspoon of $\mathrm{ZnS}(\mathrm{Ag})$ was added to about 1 teaspoon of polyurethane clear-coat paint. By weight, this mixture was: $\mathrm{ZnS}(\mathrm{Ag})=3.3062$ grams; latex = 10.0104 grams. The resultant paint mixture was thinned slightly by adding de-ionized (DI) water, which prevented cracking of the paint film during drying.
The paint was applied to glass microscope slides, each having a length of 2 inches, a width of 1 inch, and a thickness of 0.036 inches. Several slides were measured, with no significant difference in thickness. Several samples of the alpha-sensitive paint were made with variable thickness by applying the mixture to glass slides as shown in Figure 3. Thickness data at three longitudinal locations along the length of five samples is summarized below:

- CLR COAT - 1: 0.041, 0.043 and 0.043 inches; average $=0.0423$ inches $(160 \mu \mathrm{m})$.

- CLR COAT - 2: $0.045,0.047$ and 0.043 inches; average $=0.0450$ inches $(229 \mu \mathrm{m})$.

- CLR COAT - 3: 0.048, 0.048 and 0.048 inches; average $=0.0480$ inches $(305 \mu \mathrm{m})$.

- CLR COAT - 4: 0.049, 0.051 and 0.054 inches; average $=0.0513$ inches $(389 \mu \mathrm{m})$.

- CLR COAT - 5: 0.052, 0.054 and 0.058 inches; average $=0.0547$ inches $(475 \mu \mathrm{m})$.

To qualitatively determine the count rate in the absence of the polyurethane clear-coat paint, $\mathrm{ZnS}(\mathrm{Ag})$ particles were held on the surface of a glass slide by a thin layer of clear double-stick tape manufactured by 3M Corporation (Scotch Poster Tape 109). These samples are simply denoted $\mathrm{ZnS}(\mathrm{Ag})$.

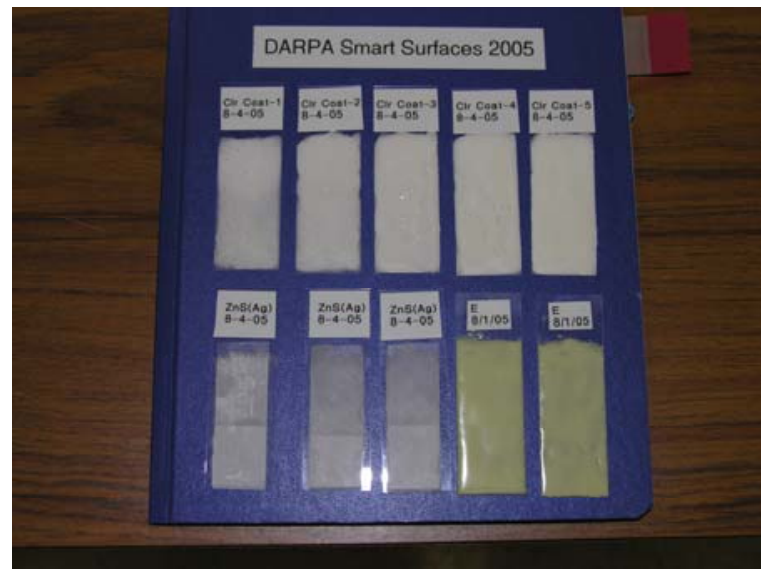

Figure 3 - Surfaces coated with scintillating paints. In this specific case, the pigment used in the paint is Ag-doped ZnS, which scintillates when bombarded by alpha particles.

This particular paint proved insensitive to all sources except the Pu-239 alpha source. The $\mathrm{ZnS}(\mathrm{Ag})$ pigment provided very good scintillation in the presence of alpha radiation, with very little phosphorescence due to exposure to ambient light. In sharp contrast, the phosphorescence from $\mathrm{ZnS}(\mathrm{Cu})$ pigments is so strong that no scintillation can be measured due to light-induced phosphorescence. 
A water-based latex paint with $\mathrm{ZnS}(\mathrm{Ag})$ scintillation pigment enabled the detection of nearsurface alpha particles from a $\mathrm{Pu}-239$ source, as shown in Table 3, and Figures 4 and 5. Alphas could be detected as long as the source was located within a distance of 2 centimeters from the painted surface, a distance determined by the penetration distance of alpha particles in air. For cases where the painted surface is located between the alpha source and the detector, an optimum paint thickness was determined to be approximately $229 \mu \mathrm{m}$.

Parametric studies were performed, determining the scintillation rate as a function of coating thickness and the distance of separation between the coating and source. An optimum paint thickness was identified. The paint has to be thick enough to provide an easily detectable level of scintillation, but not so thick that the scintillations undergo self-absorption by the paint before reaching the detector.

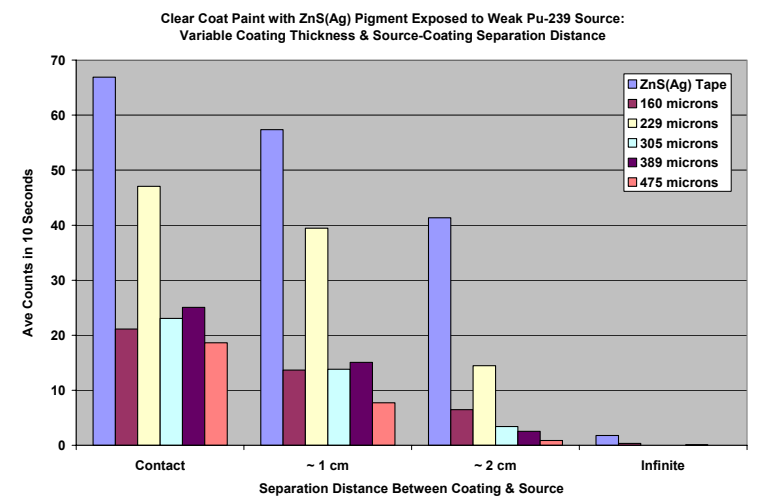

Figure 4 - The average number of counts measured during a 10-second period (average count rate) while irradiating the painted slides with a weak Pu-239 source. As expected, the count rate decreased as the distance between the source and paint increased. The optimum paint thickness for this geometry was determined to be $229 \mu \mathrm{m}$.

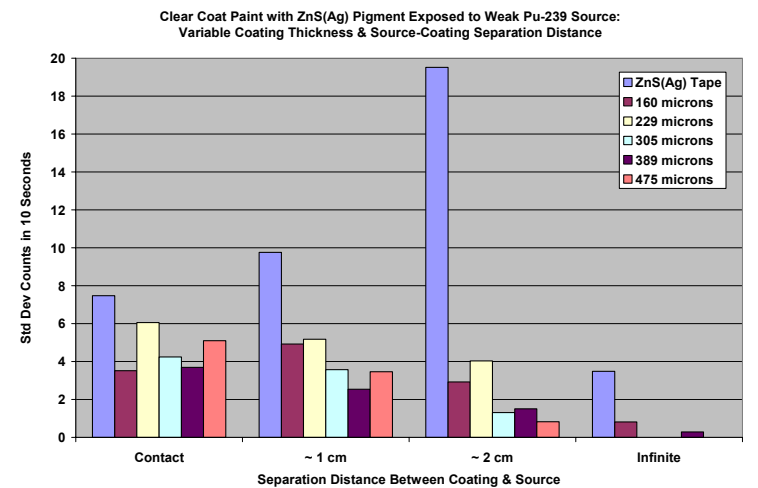

Figure 5 - Standard deviation values corresponding to the averages shown in the previous figure are summarized here.
All instrumentation was assembled and used in Building 379 at Lawrence Livermore National Laboratory (LLNL). This is a specially designed building, with unusually low levels of background radiation from terrestrial and cosmic sources. For example, the ceiling of the building is shielded. Equipment is shown in Figures 6 through 8 .

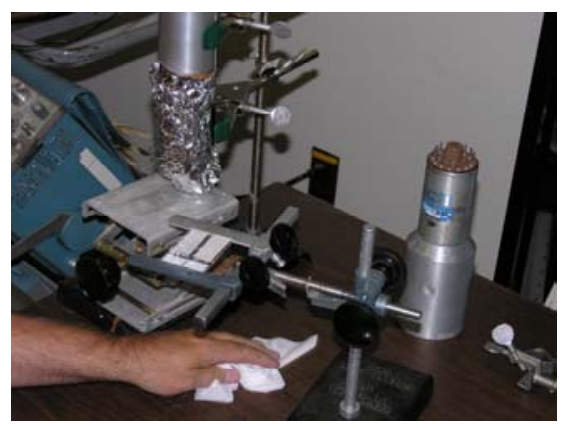

Figure 6-The painted glass slides were held in close proximity to the face of a standard photomultiplier tube (PMT), with the glass slide facing the PMT, and the exposed paint film facing the Pu-239 alpha source.

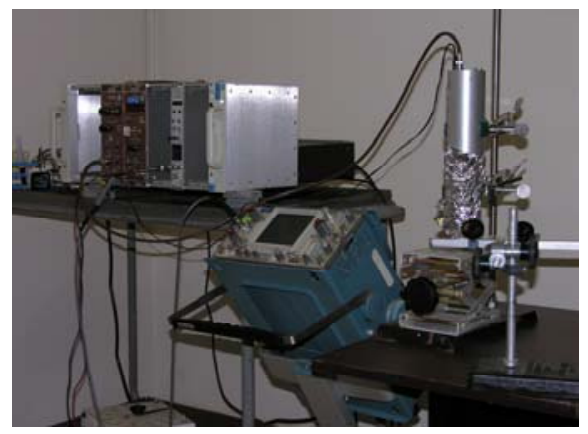

Figure 7 - Scintillations produced within the paint film by the alpha source were detected with the PMT, passed through a pre-amplifier on the base of the PMT tube, counted by a pulse counting network.

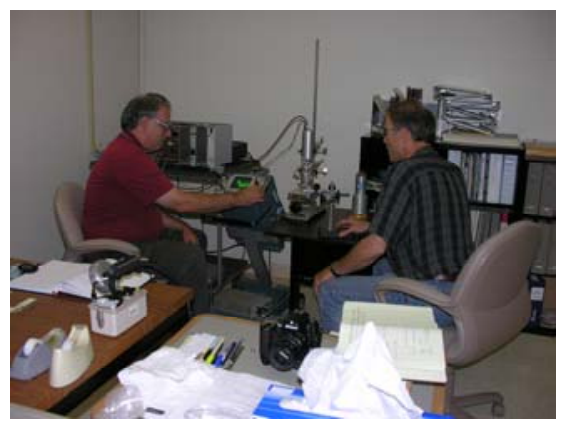

Figure 8 - This photograph shows nuclear chemist James Brunk using the oscilloscope to validate the PMT signal prior to pulse counting. 
A standard photo-multiplier tube (PMT) was used to detect scintillations emitted during irradiation of the paint film with alpha particles from the $\mathrm{Pu}-239$ source. Initial amplification of the current pulses from the PMT was done with a Video Optics Model RPS 2000 Preamplifier, which was incorporated into the base of the PMT, which also provided high-voltage to the tube.

The signal from the pre-amplifier was then input to an Ortec Model 372 Spectroscopy Amplifier. The amplified output from this module was then input to an Ortec Model 770 Pulse Counter, or to a Tennelec Model TC536 Counter-Timer, which were both read manually as the distance between the source and paint film was varied. This data acquisition system will be automated in the future.

Prior to making measurements with the paint film and PMT, the operation of the pulse-counting network was tested a standard $\mathrm{NaI}(\mathrm{Tl})$ scintillation detector for gamma detection (Harshaw Serial Number 432) and the $10-\mu \mathrm{Ci}$ Cs-137 gamma source (Serial Uumber GL732). A Tektronix digital oscilloscope was used to simultaneously monitor output from the PMT preamplifier, thereby validating the signal. After validation of the PMT signal with the oscilloscope, and optimization of the pulse counting network, measurements were made with the following instrument settings:

Video Optics Model RPS 2000 Preamplifier

- $\quad$ Negative Pulses

Ortec Model 372 Spectroscopy Preamplifier

- Coarse Gain: 500

- Fine Gain: Mid Range

- $\quad$ Shaping Time Constant: $0.5 \mu \mathrm{sec}$

- Negative Pulses

- Unipolar Output

Tennelec Model TC536 Counter-Timer

- Normal Setting

- Discriminator: 6

Gamma Sensitive Paint - Gamma-sensitive organic coatings (paints) were prepared by mixing the Varathane interior clear coat [16] and CsI(Tl) scintillation pigments, which were prepared by grinding CsI(Tl) plates. In a manner similar to that described for the preparation of water-based paints $\mathrm{ZnS}(\mathrm{Ag})$, these organic-based paints with $\mathrm{CsI}(\mathrm{Tl})$ were also applied to 1 -inch $\times 2$-inch glass slides.

As previously discussed, gamma-sensitive organic coatings (paints) were prepared by mixing the Varathane interior clear coat and CsI(Tl) scintillation pigments, which were prepared by grinding CsI(Tl) plates. These paints were then applied to glass slides, and placed on the surface of a $100-\mu \mathrm{Ci} \mathrm{Ra}-226$ gamma source. A Nikon 2DX 9-megapixel digital camera (5.4 $\mu \mathrm{m}$ pixel size) was then used to image scintillation from the painted surface during relatively brief ( 5 minute) time-lapse exposures.

A Nikon 2DX 12.8-megapixel digital camera and improvised dark box are shown in Figure 9a and 9b. A painted sample suspended in a polyethylene bag, and handing in front of $100-\mu \mathrm{Ci} \mathrm{Ra}-226$ gamma source, is shown in frames (c) and (d). The lead bricks used for shielding are also evident. Images of the scintillating pigment material and radiation-sensitive paint were collected with the digital camera are shown in frames (e) and (f), respectively. The upper frame (e) shows the scintillation from a thin sheet of $\mathrm{CsI}(\mathrm{Tl})$, and the bottom frame shows the scintillation from an organic paint with $\mathrm{CsI}(\mathrm{Tl})$ pigment. Timelapse photography with a commercially-available 12.8-megapixel camera have clearly shown radiationinduced scintillation from an early organic-based paint with ground $\mathrm{CsI}(\mathrm{Tl})$ pigment exposed to a 100 $\mu \mathrm{Ci}$ gamma source.
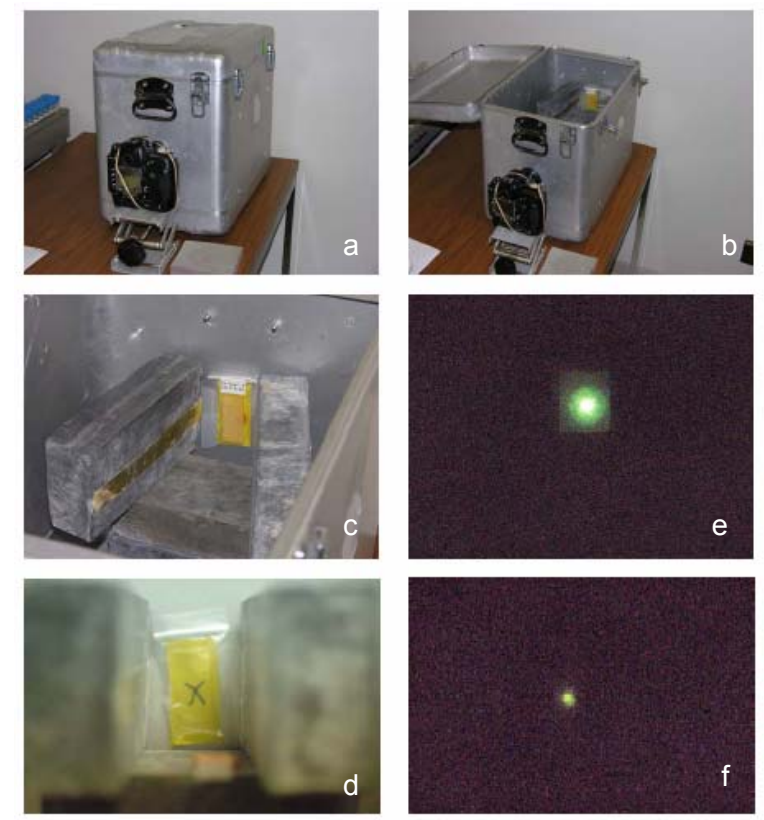

Figure 9 - Digital camera used to image surface painted with radiation-sensitive paint. The Nikon $2 D X$ 12.8-megapixel digital camera and improvised dark box are shown in frames (a) and (b). A painted sample suspended in a polyethylene bag, and handing in front of 100- $\mu \mathrm{Ci}$ Ra-226 gamma source, is shown in frames (c) and (d). The lead bricks used for shielding are also evident. Images of the scintillating pigment material and radiation-sensitive paint were collected with the digital camera are shown in frames (e) and (f), respectively. The upper frame (e) shows the scintillation from a thin sheet of $C s I(T l)$, and the bottom frame shows the scintillation from an organic paint with CsI(Tl) pigment. 
Table 3 - Data from Detection of Pu-239 with Smart Surface

ZnS(Ag) Pigment in Clear Coat with Weak Pu-239 Alpha Source

\section{Count Rate (Counts Per 10-Second Interval) - Average Values}

\begin{tabular}{|l|c|c|c|c|c|}
\hline Coating & Thickness $(\mu \mathrm{m})$ & \multicolumn{3}{|c|}{ Source-Coating Separation Distance } \\
\hline & & Contact & $\sim 1 \mathrm{~cm}$ & $\sim 2 \mathrm{~cm}$ & Infinite \\
\hline ZnS(Ag) Tape & ZnS(Ag) Tape & 66.9 & 57.3 & 41.3 & 1.8 \\
\hline $\mathrm{ZnS}(\mathrm{Ag})$ Clear Coat 1 & $160 \mu \mathrm{m}$ & 21.1 & 13.7 & 6.5 & 0.3 \\
\hline $\mathrm{ZnS}(\mathrm{Ag})$ Clear Coat 2 & $229 \mu \mathrm{m}$ & 47.1 & 39.4 & 14.5 & 0.0 \\
\hline $\mathrm{ZnS}(\mathrm{Ag})$ Clear Coat 3 & $305 \mu \mathrm{m}$ & 23.1 & 13.8 & 3.4 & 0.0 \\
\hline $\mathrm{ZnS}(\mathrm{Ag})$ Clear Coat 4 & $389 \mu \mathrm{m}$ & 25.1 & 15.1 & 2.5 & 0.1 \\
\hline $\mathrm{ZnS}(\mathrm{Ag})$ Clear Coat 5 & $475 \mu \mathrm{m}$ & 18.6 & 7.7 & 0.9 & 0.0 \\
\hline
\end{tabular}

Count Rate (Counts Per 10-Second Interval) - Standard Deviation

\begin{tabular}{|l|c|c|c|c|c|}
\hline Coating & Thickness $(\mu \mathrm{m})$ & \multicolumn{3}{|c|}{ Source-Coating Separation Distance } \\
\hline & & Contact & $\sim 1 \mathrm{~cm}$ & $\sim 2 \mathrm{~cm}$ & Infinite \\
\hline ZnS(Ag) Tape & ZnS(Ag) Tape & 7.47 & 9.76 & 19.52 & 3.49 \\
\hline ZnS(Ag) Clear Coat 1 & $160 \mu \mathrm{m}$ & 3.52 & 4.92 & 2.93 & 0.82 \\
\hline ZnS(Ag) Clear Coat 2 & $229 \mu \mathrm{m}$ & 6.06 & 5.18 & 4.03 & 0.00 \\
\hline ZnS(Ag) Clear Coat 3 & $305 \mu \mathrm{m}$ & 4.25 & 3.57 & 1.31 & 0.00 \\
\hline ZnS(Ag) Clear Coat 4 & $389 \mu \mathrm{m}$ & 3.69 & 2.54 & 1.51 & 0.29 \\
\hline ZnS(Ag) Clear Coat 5 & $475 \mu \mathrm{m}$ & 5.10 & 3.47 & 0.83 & 0.00 \\
\hline
\end{tabular}

\section{THERMO-LUMINESCENT PAINT}

Paint Preparation - Thermo-luminescent paints were prepared with thermo-luminescent pigment produced by milling TLD-700 Li-7 fluoride ${ }^{7} \mathrm{LiF}$ ) chips, and mixing it with a clear organic paint for high-temperature applications (PJH Brands SP-115 VHT Clear Coat). The ratio of clear coat to powder was 10:4 by weight. The pigment received an initial dose of more than 20,000 rad from a Co-60 gamma source before incorporation into the paint. Paint samples identified as $\operatorname{LiF}(\# 1), \operatorname{LiF}(\# 2)$, and $\operatorname{LiF}(\# 3)$ were prepared with coarse $(-75 /+53 \mu \mathrm{m})$, medium $(-53 /+38 \mu \mathrm{m})$ and fine $(-38 \mu \mathrm{m})$ pigments, respectively.

Detection - Emissions from the coating were monitored as a function of time and temperature with a Digi-Base ${ }^{\mathrm{TM}}$ photomultiplier tube (PMT), interfaced directly to a laptop computer via the USB-2 port, as shown in Figure 10a. A Nikon D2X digital camera was also used to image thermo-luminescence of paint samples as they were heated. Figure 10b shows an image of $\mathrm{LiF}(\# 1)$ during heating. Interrogation involved heating the irradiated coatings in a controlled manner, using a hot plate. The coatings were heated to $300^{\circ} \mathrm{C}$ to $306^{\circ} \mathrm{C}$ on the surface of a 4inch $\times 4$-inch hot plate.
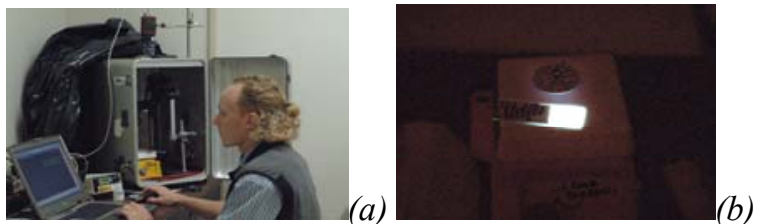

Figure 10 - The upper photograph (a) shows experimental apparatus for interrogation of thermoluminescent paints. Interrogation involved heating the irradiated coatings in a controlled manner, using a hot plate. Emissions from the coating were monitored as a function of time and temperature with a DigiBase ${ }^{T M}$ photomultiplier tube (PMT), interfaced directly to a laptop computer via the USB-2 port. The coatings can also be heated with a "stand-off laser system. The lower photograph (b) shows thermoluminescence from a coating prepared with powder prepared by milling TLD-700 Li-7 fluoride ( $\left.{ }^{7} \mathrm{LiF}\right)$ chips, and a clear organic paint for high-temperature applications (PJH Brands SP-115 VHT Clear Coat). 
First Irradiation - Time-lapse photographs were taken of $\operatorname{LiF}(\# 2)$, the paint with medium-size pigment, at 5-second intervals during heating, as shown in Figures 11a through 11c. The first image, Figure $11 \mathrm{a}$, was taken at approximately $300^{\circ} \mathrm{C}$, with intense, easily seen thermo-luminescence.

Time-lapse photographs were taken of $\operatorname{LiF}(\# 3)$, the paint with fine pigment, at 5-second intervals during heating, as shown Figures 12a through 12h. The first image, Figure 14a, was taken at 296 to $300^{\circ} \mathrm{C}$. These images show the decay in luminescence with time. After the coatings are fully annealed, they can be re-used, thus detected other gamma exposures. These time-lapse images show decreasing thermoluminescence with increasing time at temperature.

Figure 13a shows the initial temperature and thermo-luminescence versus time for $\operatorname{LiF}(\# 1)$, the paint with coarse pigment. Figure $13 \mathrm{~b}$ shows the thermo-luminescence as a function of temperature, and is based on the transient data shown in the first figure. From these data, the temperature threshold for interrogation of these gamma-sensitive paints is between 100 to $150^{\circ} \mathrm{C}\left(\sim 120^{\circ} \mathrm{C}\right)$. It is reasonable, based on this data, to expect that these paints could store information in hot desert climates.

Figure 14a shows the initial temperature and thermo-luminescence transients for $\mathrm{LiF}(\# 2)$, the paint with medium pigment. Figure $14 \mathrm{~b}$ shows the thermoluminescence as a function of temperature, and is based on the transient data shown in Figure 13a. From these data, the temperature threshold for interrogation of this paint also appears to be between 100 to $150^{\circ} \mathrm{C}$ $\left(\sim 120^{\circ} \mathrm{C}\right)$. The signal intensity is comparable to that obtained with $\mathrm{LiF}$ (\#1), prepared with coarse pigment.

Figure $15 \mathrm{a}$ shows the initial temperature and thermo-luminescence versus time for $\operatorname{LiF}(\# 3)$, the paint with fine pigment. Figure $15 \mathrm{~b}$ shows the thermo-luminescence as a function of temperature, and is based on the transient data shown in Figure 15a. From these data, the temperature threshold for interrogation of this paint also appears to be between 100 to $150^{\circ} \mathrm{C}\left(\sim 120^{\circ} \mathrm{C}\right)$. The signal intensity with this pigment less than that obtained with the coarser $(-75 /+53$ and $-53 /+38 \mu \mathrm{m})$ pigments.

Figure 16 shows a comparison of the initial thermo-luminescence as a function of temperature for $\mathrm{LiF}(\# 1), \mathrm{LiF}(\# 2)$ and $\mathrm{LiF}(\# 3)$, prepared with coarse $(-75 /+53 \mu \mathrm{m})$, medium $(-53 /+38 \mu \mathrm{m})$, and fine $(-38$ $\mu \mathrm{m})$ pigments, respectively. From these data, the temperature threshold for interrogation of these gamma-sensitive paints appears to be approximately 100 to $150^{\circ} \mathrm{C}\left(\sim 120^{\circ} \mathrm{C}\right)$, regardless of pigment size. The signal intensity is less than that obtained with the coarser $(-75 /+53$ and $-53 /+38 \mu \mathrm{m}$ fractions) thermoluminescent pigments. A slight optimum in performance may exist for the $-53 /+38 \mu \mathrm{m}$ size pigment, with the fine pigment having the poorest performance.
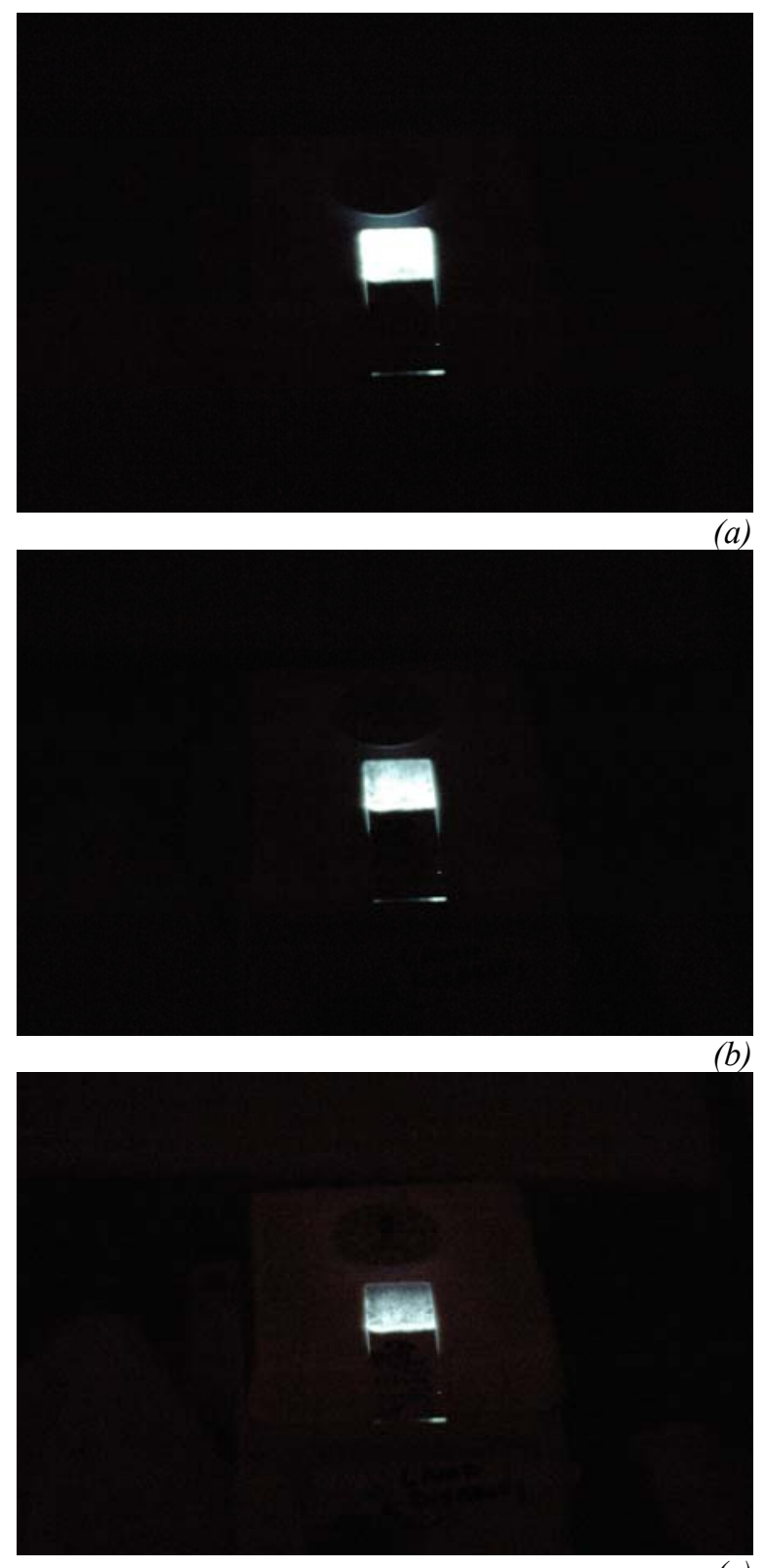

(c)

Figure 11- These three figures (a through c) are a time-lapse thermo-luminescent sequence taken at 5second intervals, with the first image (a) taken at approximately $300^{\circ} \mathrm{C}$. In this case, the coating was prepared with a finer powder, which was prepared by milling TLD-700 Li-7 fluoride ( $\mathrm{LiF}$ ) chips, taking the $-53 /+38 \mu m$ size fraction, and mixing it with a clear organic paint for high-temperature applications (PJH Brands SP-115 VHT Clear Coat). 

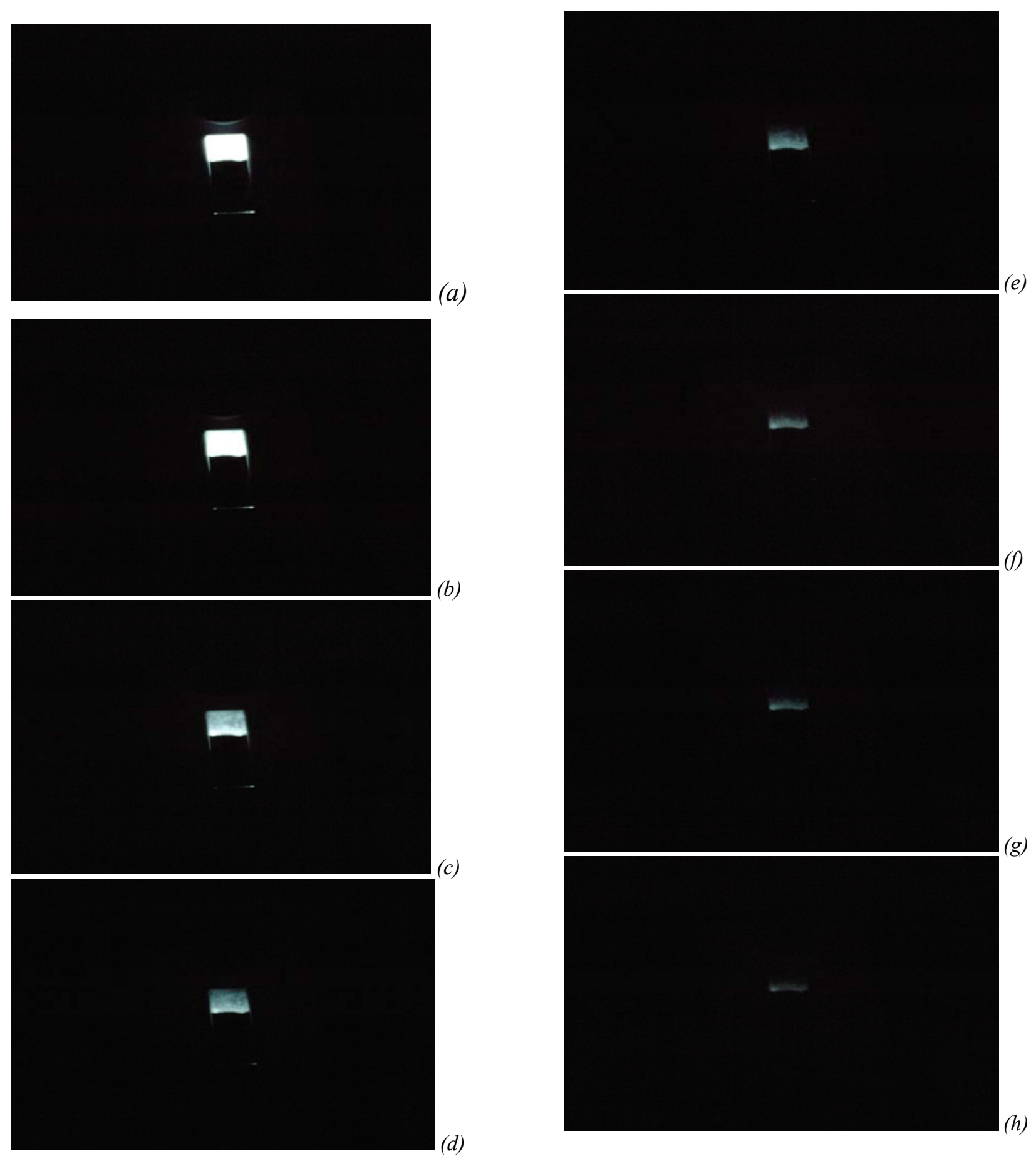

Figure 12 - These eight figures (a through $h$ ) are a time-lapse thermo-luminescent sequence taken at 5-second intervals, with the first image (a) taken at 296 to $300^{\circ} \mathrm{C}$. In this case, the coating was prepared with an extremely fine powder, which was prepared by milling TLD-700 Li-7 fluoride ( ${ }^{7}$ LiF) chips, taking the -38 um size fraction, and mixing it with a clear organic paint for high-temperature applications (PJH Brands SP-115 VHT Clear Coat). 


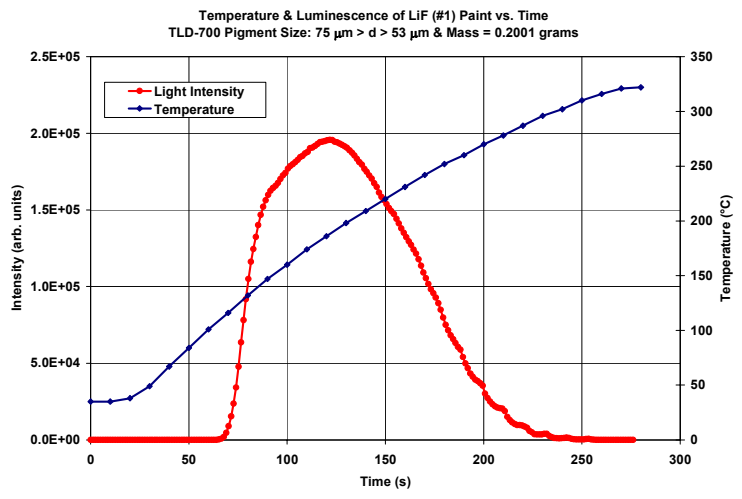

(a)

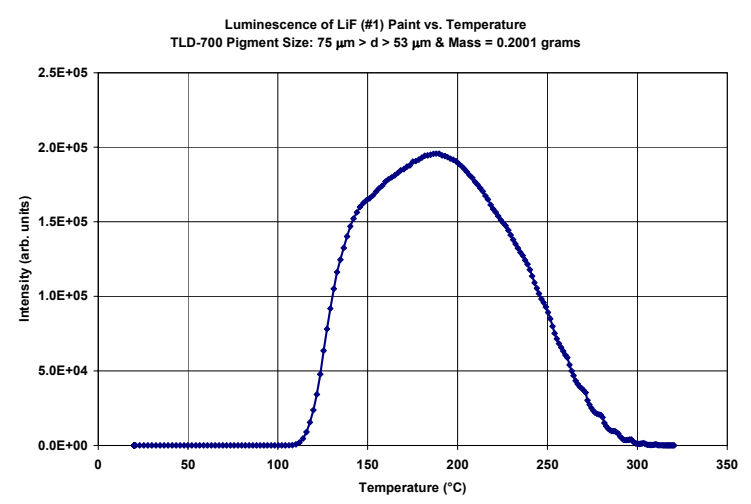

(b)

Figure 13 - The first figure (a) shows the initial temperature and thermo-luminescence transients for LiF (\#1). The second figure (b) shows the thermoluminescence as a function of temperature, and is based on the transient data shown in the first figure. This coating was prepared with relatively coarse powder, which was produced by milling TLD-700 Li7 fluoride ( $\mathrm{LiF})$ chips, and taking the $-75 /+53 \mu \mathrm{m}$ size fraction, and mixing it with a clear organic paint for high-temperature applications (PJH Brands SP115 VHT Clear Coat).

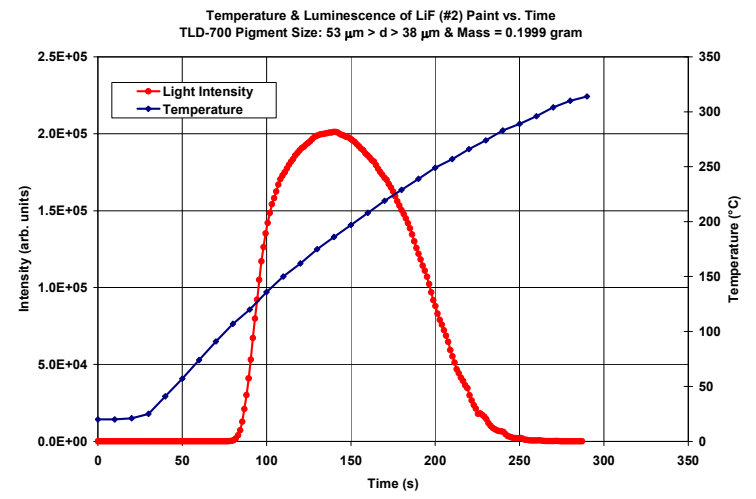

(a)

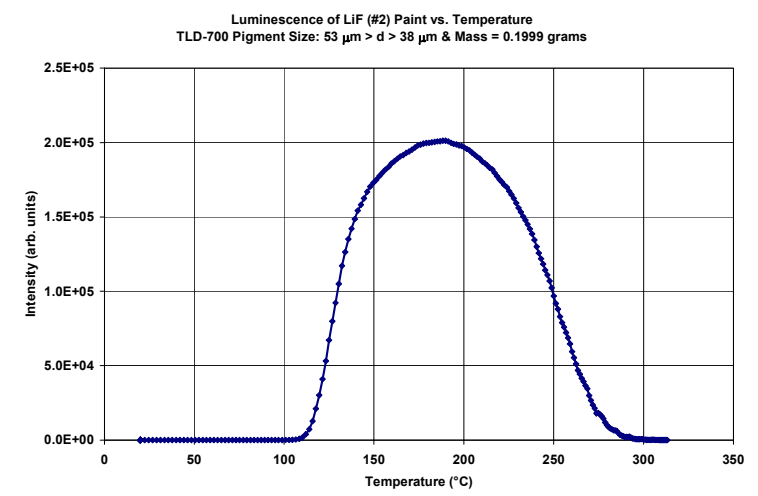

(b)

Figure 14 - The first figure (a) shows the initial temperature and thermo-luminescence transients for LiF (\#2). The second figure (b) shows the thermoluminescence as a function of temperature, and is based on the transient data shown in the first figure. In this case, the coating was prepared with a finer powder, which was prepared by milling TLD-700 Li-7 fluoride ( $\mathrm{LiF}$ ) chips, taking the $-53 /+38$ um size fraction, and mixing it with a clear organic paint for high-temperature applications (PJH Brands SP-115 VHT Clear Coat). 


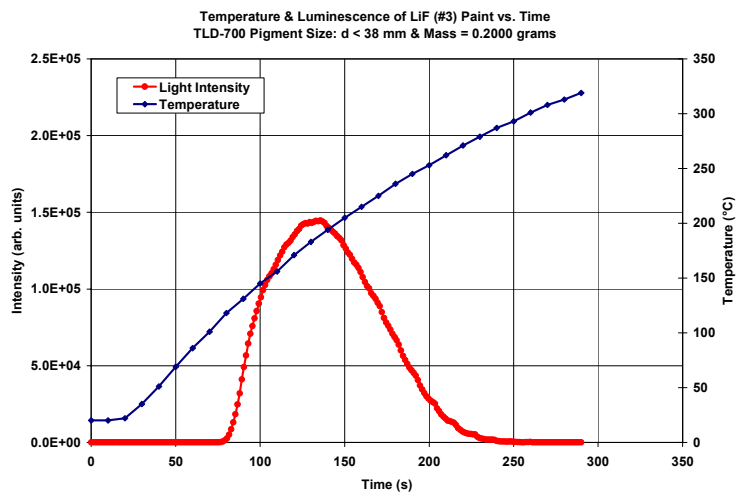

(a)

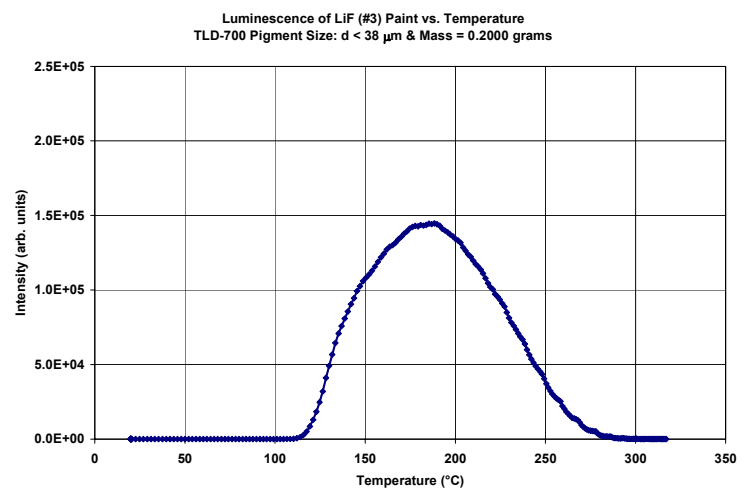

(b)

Figure 15 - The first figure (a) shows the initial temperature and thermo-luminescent transients for $\mathrm{LiF}$ (\#3). The second figure (b) shows the thermoluminescence as a function of temperature, and is based on the transient data shown in the first figure. In this case, the coating was prepared with an extremely fine powder, which was prepared by milling TLD-700 Li-7 fluoride ( $\mathrm{LiF}$ ) chips, taking the $-38 \mu \mathrm{m}$ size fraction, and mixing it with a clear organic paint for high-temperature applications (PJH Brands SP115 VHT Clear Coat).

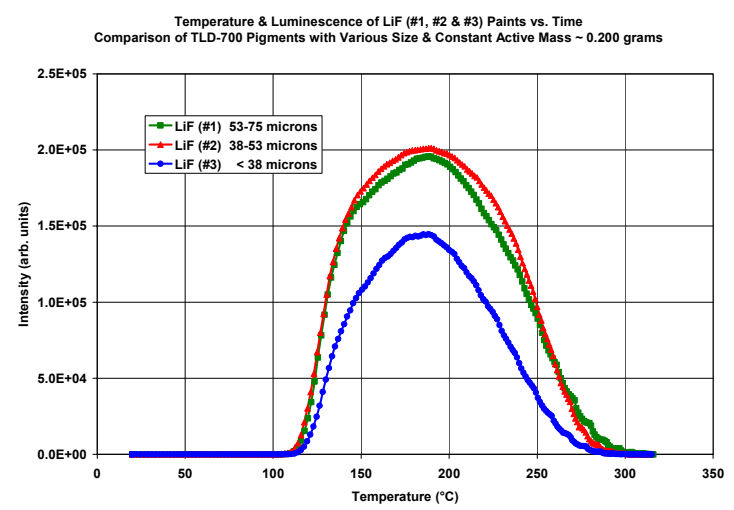

Figure 16 - The figure shows a comparison of the initial thermo-luminescence as a function of temperature for paints prepared with the three sizefraction pigments: coarse $(-75 /+53 \mu \mathrm{m})$; medium $(-53 /+38 \mu \mathrm{m})$; and fine $(-38 \mu \mathrm{m})$. These are identified as LiF (\#1), LiF (\#2) and LiF (\#3), respectively. As previously discussed, each coating was prepared with powder that was produced by milling TLD-700 Li-7 fluoride ( LiF) chips, taking the desired size fraction, and mixing it with a clear organic paint for high-temperature applications (PJH Brands SP-115 VHT Clear Coat). 
Second Irradiation - As previously discussed, thermo-luminescent paints were made with the three size-fraction pigments: coarse $(-75 /+53 \mu \mathrm{m})$; medium $(-53 /+38 \mu \mathrm{m})$; and fine $(-38 \mu \mathrm{m})$. These paints were identified as $\operatorname{LiF}(\# 1), \operatorname{LiF}(\# 2)$ and $\operatorname{LiF}(\# 3)$, respectively. These paints were thermally reset after initial irradiation by heating to a temperature above $300^{\circ} \mathrm{C}$. The samples were then irradiated a second time with a dose of approximately 50,000 rad and interrogated. Figures 17, 18 and 19 show the temperature and thermo-luminescence transients following the second irradiation for $\operatorname{LiF}(\# 1), \operatorname{LiF}(\# 2)$ and $\mathrm{LiF}(\# 3)$, respectively.

Figure 20 shows a comparison of the thermoluminescence as a function of temperature for paint samples This figure shows the thermo-luminescent response verses temperature following the second irradiation. From these data, the temperature threshold for interrogation of these gamma-sensitive paints appears to be approximately 80 to $100^{\circ} \mathrm{C}\left(\sim 85^{\circ} \mathrm{C}\right)$. In comparison to the initial measurement, the threshold temperature for the onset of thermo-luminescence may have shifted to lower levels, but requires additional work to verify. The signal intensity from the fine pigments $(-38 \mu \mathrm{m})$ is less than that obtained with the coarser pigments $(-75 /+53$ and $-53 /+38 \mu \mathrm{m}$ fractions), which is consistent with the initial irradiation. It also appears that a slight optimum in performance may exist for the $-53 /+38 \mu \mathrm{m}$ size pigment, with the fine pigment having inferior performance.
Third Irradiation - Thermo-luminescent paint samples $\operatorname{LiF}(\# 1), \operatorname{LiF}(\# 2)$ and $\operatorname{LiF}(\# 3)$ were irradiated a third time with a dose of $\sim 5000 \mathrm{rad}$, as shown in Figures 21 through 23. This figure shows the temperature and thermo-luminescent transients following the third irradiation. Figure 24 shows a comparison of the thermo-luminescence as a function of temperature for these three paint samples, $\operatorname{LiF}(\# 1)$, $\mathrm{LiF}(\# 2)$ and $\operatorname{LiF}(\# 3)$, obtained after each was irradiated a third time with a dose of $\sim 5000 \mathrm{rad}$. These samples were prepared with three size-fraction pigments, coarse $(-75 /+53 \mu \mathrm{m})$, medium $(-53 /+38$ $\mu \mathrm{m})$ and fine $(-38 \mu \mathrm{m})$, respectively.

The temperature threshold for interrogation is between 120 and $150^{\circ} \mathrm{C}$ at a lower dose of $5000 \mathrm{rad}$, and is greater than the threshold of 80 to $100{ }^{\circ} \mathrm{C}$ observed with the higher dose of 50,000 rad. It is therefore concluded that the stored dose information is more stable as the dose decreases.

From the comparison of thermo-luminescence data in Figures 25 and 26, it can be concluded that the performance of pigments in $\mathrm{LiF}(\# 1)$ and $\mathrm{LiF}(\# 2)$ are comparable, whereas the performance of pigment in $\mathrm{LiF}$ (\#3) is relatively poor. The size distribution of pigments used in $\operatorname{LiF}(\# 1)$ and $\operatorname{LiF}(\# 2)$ were coarse $(-75 /+53 \mu \mathrm{m})$ and medium $(-53 /+38 \mu \mathrm{m})$, respectively. The size distribution of the pigment used in $\operatorname{LiF}(\# 3)$ was fine $(-38 \mu \mathrm{m})$. It is therefore concluded that radiation sensitive paints using this type of TL pigment should use particle sizes greater than $38 \mu \mathrm{m}$. The finer pigments may have too many defects from grinding to function well as thermoluminescent micro crystals.

Figure 27 shows the integrated response of TL paint sample $\operatorname{LiF}(\# 2)$, with two points obtained by integrating the responses represented by Figure 26 . Easily visible detection is possible at exposures below $1000 \mathrm{rad}$, and with enhanced paints and detection instruments, detection well below 1 rad should be possible. However, the lower detection level requires additional development.

As shown in Figure 28, the peak emission during interrogation is at a wavelength of $400 \mathrm{~nm}$, in the violet-to-ultraviolet range, which was expected. 


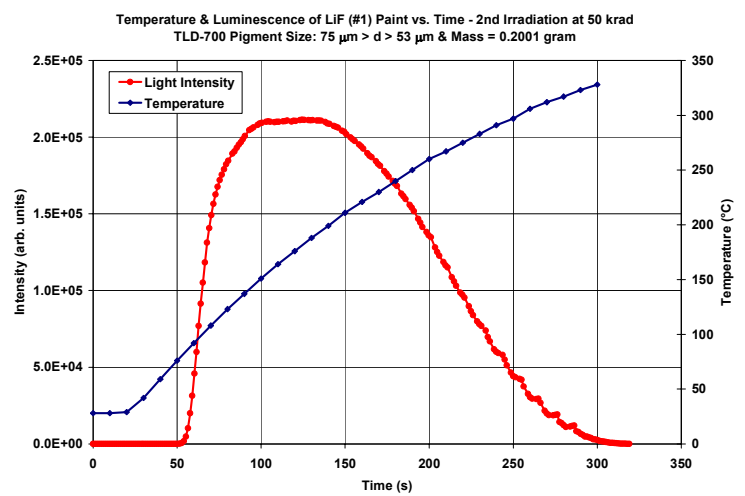

Figure 17 - TL paint sample LiF (\#1) was prepared by mixing the $-75 /+53 \mu \mathrm{m}$ size fraction of ${ }^{7} \mathrm{LiF}$ powder (TLD-700 Li-7 fluoride) with a clear organic paint for high-temperature applications (PJH Brands SP-115 VHT Clear Coat). The sample was irradiated a second time with a dose of $\sim 50,000 \mathrm{rad}$ and interrogated. This figure shows the temperature and thermo-luminescent transients following the second irradiation.

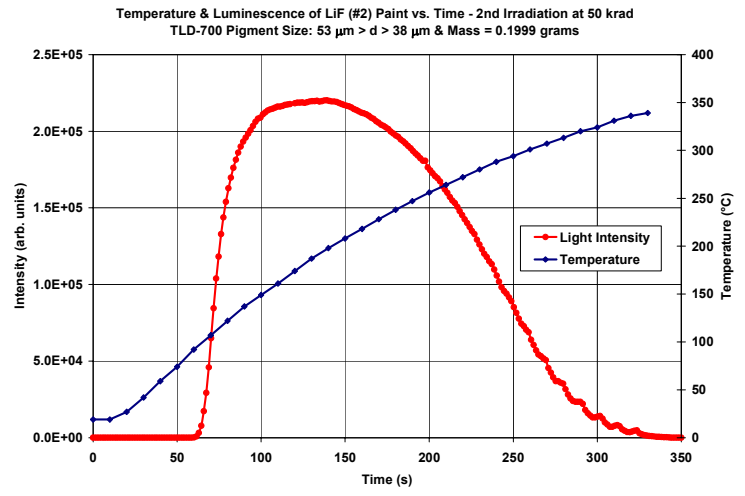

Figure 18 - TL paint sample LiF (\#2) was prepared by mixing the $-53 /+38 \mu \mathrm{m}$ size fraction of ${ }^{7} \mathrm{LiF}$ powder (TLD-700 Li-7 fluoride) with a clear organic paint for high-temperature applications (PJH Brands SP-115 VHT Clear Coat). This sample was irradiated a second time with a dose of $\sim 50,000 \mathrm{rad}$ and interrogated. This figure shows the temperature and thermo-luminescent transients following the second irradiation.

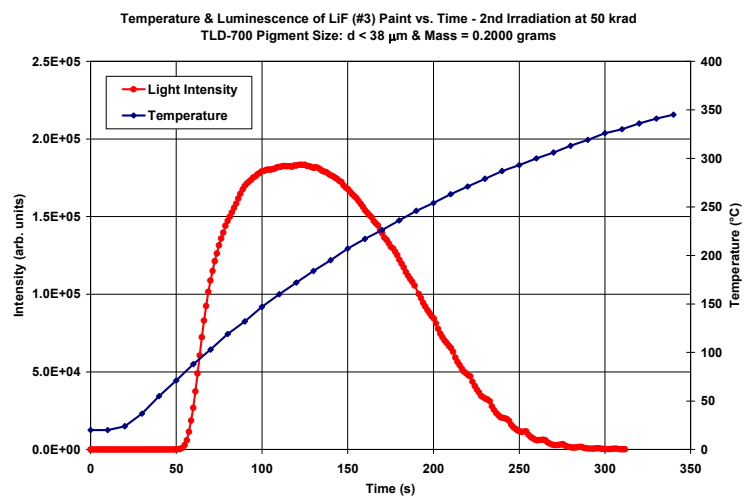

Figure 19 - TL paint sample LiF (\#3) was prepared by mixing the $-38 \mu \mathrm{m}$ size fraction of ${ }^{7} \mathrm{LiF}$ powder (TLD-700 Li-7 fluoride) with a clear organic paint for high-temperature applications (PJH Brands SP-115 VHT Clear Coat). This sample was irradiated a second time with a dose of $\sim 50,000$ rad and interrogated. This figure shows the temperature and thermo-luminescent transients following the second irradiation.

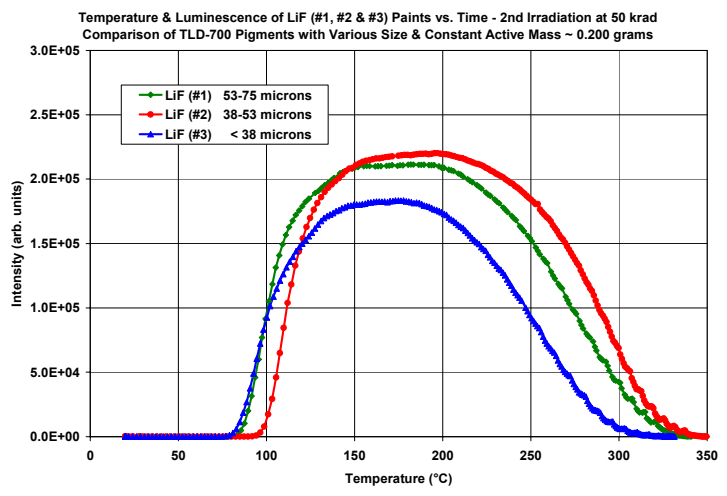

Figure 20 - The figure shows a comparison of the thermo-luminescence as a function of temperature for paints made with the three size-fraction pigments: coarse $(-75 /+53 \mu \mathrm{m}) ;$ medium $(-53 /+38 \mu \mathrm{m})$; and fine $(-38 \mu \mathrm{m})$. Samples were prepared by mixing various size fractions of ${ }^{7}$ LiF powder with a clear organic paint for high-temperature applications. Each sample was irradiated a second time with a dose of $\sim 50,000 \mathrm{rad}$ and interrogated. This figure shows the thermo-luminescent response verses temperature following the second irradiation. 


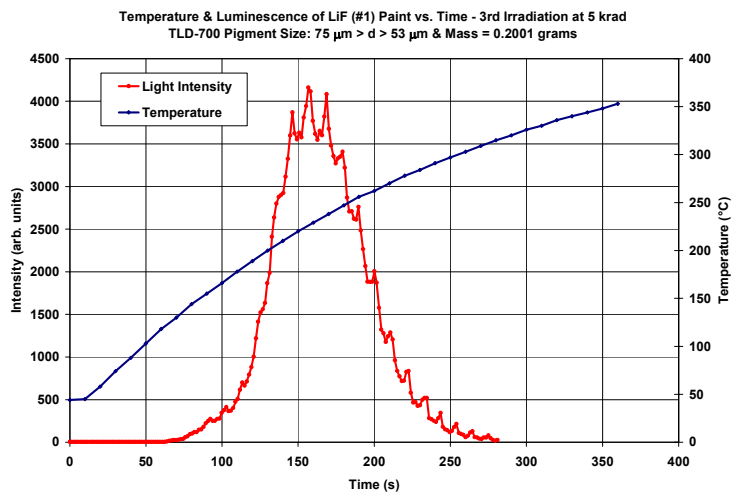

Figure 21 - TL paint sample LiF (\#1) was prepared by mixing the $-75 /+53 \mu \mathrm{m}$ size fraction of ${ }^{7} \mathrm{LiF}$ powder (TLD-700 Li-7 fluoride) with a clear organic paint for high-temperature applications (PJH Brands SP-115 VHT Clear Coat). The sample was irradiated a third time with a dose of $\sim 5000 \mathrm{rad}$ and interrogated. This figure shows the temperature and thermo-luminescent transients following the second irradiation.

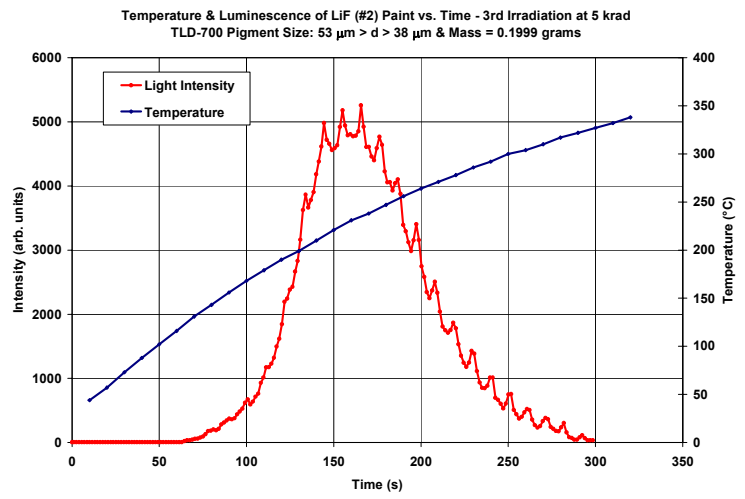

Figure 22 - TL paint sample LiF (\#2) was prepared by mixing the $-53 /+38 \mu m$ size fraction of ${ }^{7} \mathrm{LiF}$ powder (TLD-700 Li-7 fluoride) with a clear organic paint for high-temperature applications (PJH Brands SP-115 VHT Clear Coat). This sample was irradiated a third time with a dose of $\sim 5000 \mathrm{rad}$ and interrogated. This figure shows the temperature and thermo-luminescent transients following the second irradiation.

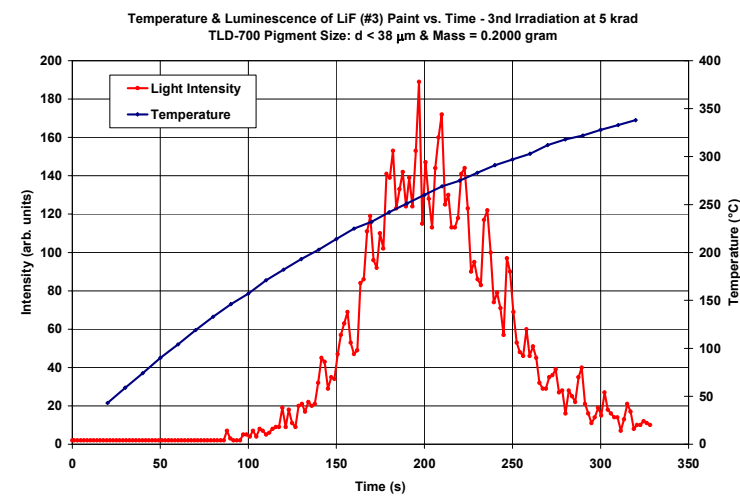

Figure 23 - TL paint sample LiF (\#3) was prepared by mixing the $-38 \mu \mathrm{m}$ size fraction of ${ }^{7} \mathrm{LiF}$ powder (TLD-700 Li-7 fluoride) with a clear organic paint for high-temperature applications (PJH Brands SP-115 VHT Clear Coat). This sample was irradiated a third time with a dose of $\sim 5000$ rad and interrogated. This figure shows the temperature and thermo-luminescent transients following the second irradiation.

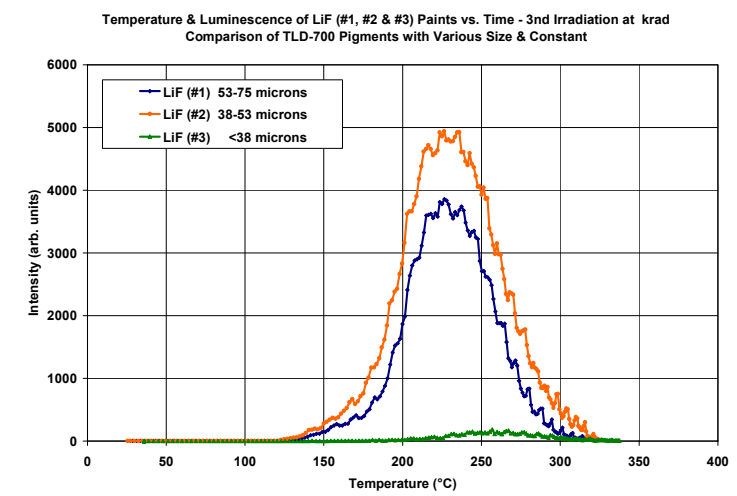

Figure 24 - The figure shows a comparison of the thermo-luminescence as a function of temperature for paints made with the three size-fraction pigments: coarse $(-75 /+53 \mu \mathrm{m})$; medium $(-53 /+38 \mu \mathrm{m})$; and fine $(-38 \mu \mathrm{m})$. Samples were prepared by mixing various size fractions of ${ }^{7} \mathrm{LiF}$ powder with a clear organic paint for high-temperature applications. Each sample was irradiated a third time with a dose of $\sim 5000 \mathrm{rad}$ and interrogated. This figure shows the thermo-luminescent response verses temperature following the second irradiation. 


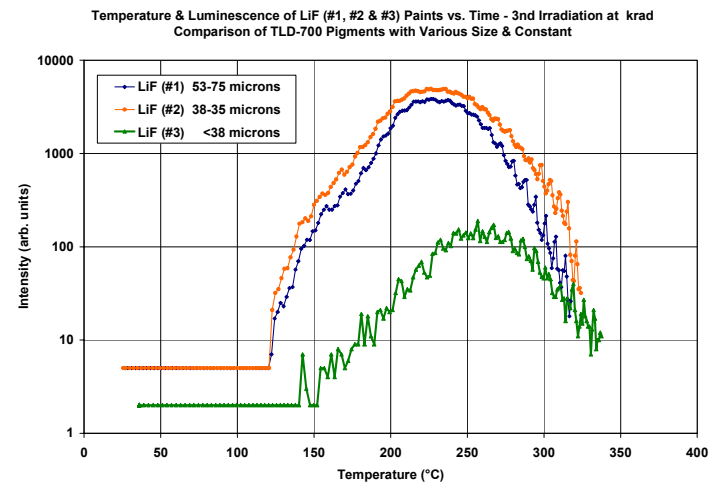

Figure 25 - The figure shows a comparison of the thermo-luminescence as a function of temperature for paints made with the three size-fraction pigments: coarse $(-75 /+53 \mu \mathrm{m})$; medium $(-53 /+38 \mu \mathrm{m})$; and fine $(-38 \mu \mathrm{m})$. Samples were prepared by mixing various size fractions of ${ }^{7} \mathrm{LiF}$ powder with a clear organic paint for high-temperature applications. Each sample was irradiated a third time with a dose of 5000 rad and interrogated. This figure shows the thermo-luminescent response verses temperature following the second irradiation.

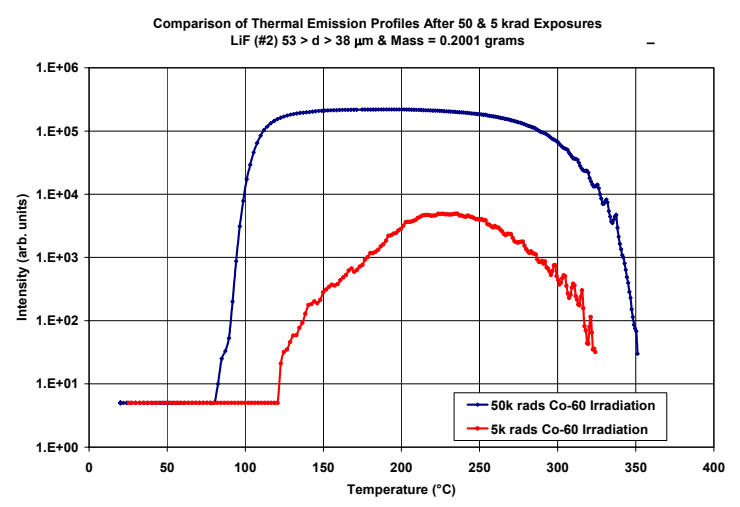

Figure 26 - TL paint sample LiF (\#2) was prepared by mixing the $-53 /+38 \mu m$ size fraction of ${ }^{7} \mathrm{LiF}$ powder (TLD-700 Li-7 fluoride) with a clear organic paint for high-temperature applications (PJH Brands SP-115 VHT Clear Coat). This sample was irradiated three times, a first and second time with a dose of $\sim 50,000 \mathrm{rad}$, and a third time with a dose of $\sim 5000$ rad. This figure shows the effect of dose on thermoluminescence.

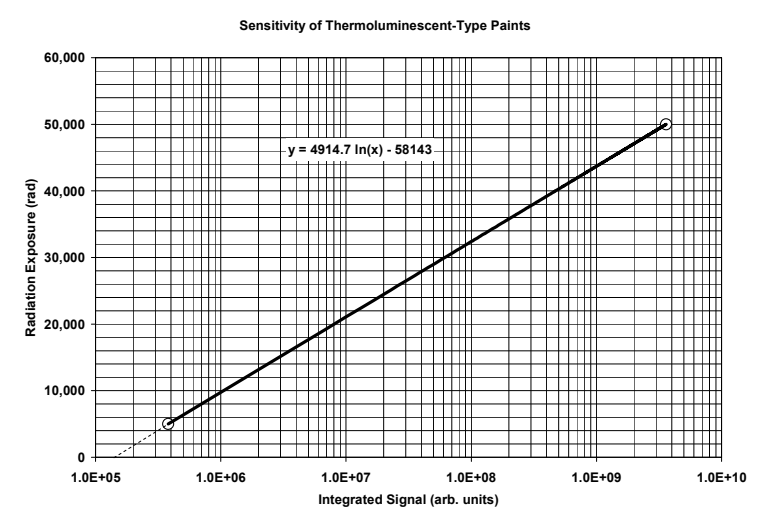

Figure 27 - This figure shows the integrated response of TL paint sample LiF (\#2), with two points obtained by integrating the responses represented by Figure 26. Easily visible detection is possible at exposures below 1000 rad, and with enhanced paints and detection instruments, detection well below 1 rad should be possible. However, the lower detection level requires additional development.

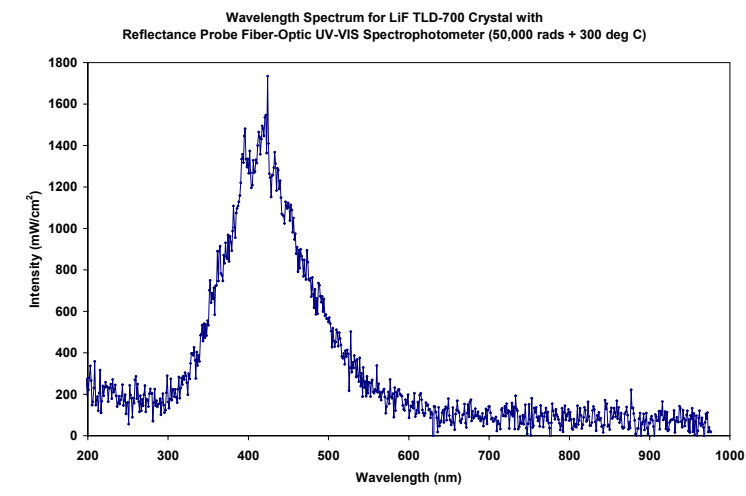

Figure 28 - Thermo-luminescence spectrum for LiF TLD-700 crystal obtained at $300{ }^{\circ} \mathrm{C}$ with fiber optic probe and ultraviolet-visible spectrophotometer after receiving 70,000 rad exposure with Co-60 gamma source. 


\section{CONCLUSIONS}

A simple water-based optically-transparent latex paint with $\mathrm{ZnS}(\mathrm{Ag})$ scintillation pigment produced a paint with opaque appearance, and enabled the detection of near-surface alpha particles from a very weak ( $1 \mathrm{nCi}) \mathrm{Pu}-239$ source. Alphas could be detected as long as the source was located within a distance of 2 centimeters, a distance determined by the penetration distance of alpha particles in air. For cases where the painted surface is located between the alpha source and the detector (PMT with pulse counting network) an optimum paint thickness was determined to be approximately $229 \mu \mathrm{m}$.

Time-lapse photography with a commerciallyavailable 12.8-megapixel camera have clearly shown radiation-induced scintillation from an early organicbased paint with ground $\mathrm{CsI}(\mathrm{Tl})$ scintillation pigment exposed to a $100-\mu \mathrm{Ci} 0.26-\mathrm{MeV}$ gamma source. This simple and elegant approach provides a powerful detection capability with profound implications.

Radiation sensitive paints capable of integrating dose were prepared with the three size-fraction TLtype pigments: coarse $(-75 /+53 \mu \mathrm{m})$; medium $(-53 /+38 \mu \mathrm{m})$; and fine $(-38 \mu \mathrm{m})$. As previously discussed, each coating was prepared with powder that was produced by milling TLD-700 Li-7 fluoride $\left.{ }^{7} \mathrm{LiF}\right)$ chips, taking the desired size fraction, and mixing it with a clear organic paint for hightemperature applications (PJH Brands SP-115 VHT Clear Coat). In all cases, the ratio of clear coat to powder was $10: 4$ by weight. First, the coating pigment was given a dose of $>20,000$ rad from a Co60 gamma source, mixed with the clear coat paint, and then applied to a surface. The surface was then heated with a hotplate while the thermo-luminescent response was monitored with a photomultiplier tube. From the data obtained with these gamma-sensitive paints, the temperature threshold for interrogation was determined to be approximately 110 to $120^{\circ} \mathrm{C}$. The signal intensity is less than that obtained with the coarser $(-75 /+53$ and $-53 /+38 \mu \mathrm{m}$ fractions) thermoluminescent pigments. A slight optimum in performance may exist for the $-53 /+38 \mu \mathrm{m}$ size pigment, with the fine pigment having inferior performance. Each sample was then irradiated a second time, with a dose of $\sim 50,000 \mathrm{rad}$, and interrogated. The thermo-luminescent response was measured as a function of temperature following the second irradiation. From these data, the temperature threshold for interrogation of these gamma-sensitive paints appears to be approximately 80 to $100^{\circ} \mathrm{C}$. In comparison to the initial measurement, the threshold temperature for the onset of thermo-luminescence may have shifted to lower levels, but requires additional work to verify. The signal intensity from the fine pigments $(-38 \mu \mathrm{m})$ is less than that obtained with the coarser pigments $(-75 /+53$ and $-53 /+38 \mu \mathrm{m}$ fractions), which is consistent with the initial irradiation. It also appears that a slight optimum in performance may exist for the $-53 /+38 \mu \mathrm{m}$ size pigment, with the fine pigment having inferior performance. In either case paint has been developed that is clearly capable of integrating gamma dose, and allowing interrogation upon heating to a modest temperature above ambient.

A third irradiation of TL-type paints delivered a dose of approximately $5000 \mathrm{rad}$. In this case, the temperature threshold for interrogation in this case was between 120 and $150^{\circ} \mathrm{C}$, and was greater than the threshold of 80 to $100{ }^{\circ} \mathrm{C}$ observed with the higher dose of 50,000 rad. It is therefore concluded that the stored dose information is more stable as the dose decreases.

It was concluded that the performance of pigments in $\mathrm{LiF}(\# 1)$ and $\mathrm{LiF}(\# 2)$ were comparable, whereas the performance of pigment in $\operatorname{LiF}(\# 3)$ was relatively poor. The size distribution of pigments used in $\operatorname{LiF}(\# 1)$ and $\operatorname{LiF}(\# 2)$ were coarse $(-75 /+53 \mu \mathrm{m})$ and medium $(-53 /+38 \mu \mathrm{m})$, respectively. The size distribution of the pigment used in $\operatorname{LiF}(\# 2)$ was fine $(-38 \mu \mathrm{m})$. It was therefore concluded that radiation sensitive paints using this type of TL pigment should use particle sizes greater than $38 \mu \mathrm{m}$ s. The finer pigments may have had too many defects from grinding to function well as thermo-luminescent micro crystals. The peak emission during interrogation is at a wavelength of $400 \mathrm{~nm}$, in the violet-to-ultraviolet range, which was expected.

\section{DISCUSSION}

While these paint films will never compete with high-efficiency solid-state radiation detectors in regard to sensitivity, these paints could be developed to the point where they have performance comparable to standard film badges. Unlike either expensive solid-state detectors or discrete film badges, these paints can be used for covering large areas such as the walls, ceilings and floors of structures, thereby providing potential advantages for decontamination of surfaces covered with widespread radioactive contamination. Other potential advantages of this radiation-sensitive paint technology could include the tagging and labeling of containers used for the transportation and storage of nuclear materials, and for the tagging and labeling of piping, vessels and other process equipment used for the processing and reprocessing of nuclear materials, including but not limited to spent nuclear fuel.

Radiation-sensitive paints and coatings could be used to monitor exposure in various scenarios of interest: (1) as paints for buildings and equipment in industrial plants involved in the production of nuclear and radiological materials; (2) as paints for the inside of nuclear power plants, nuclear powered ships, and 
submarines; (3) as paints for trucks and shipping containers and road-side facilities along shipping routes; (5) as paints for unmanned aerial vehicles, micro airships, and other surveillance devices; and (6) as paints for the detection and monitoring of activities involving radiological materials.

In addition to enabling the long-term exposure (dose) of operating personnel in nuclear plants and nuclear-powered ships to be monitored, surfaces coated with these paints could be used to track and image the spread of radioactive contamination. Ultimately, thermo-luminescent paints and coatings could be used as a basis for qualifying the receipt of shipping containers for acceptance into the United States, where such qualification could be done through field interrogation of the painted surface, or through quantification of sampled paint chips. These coatings can also be used on aircraft, to detect corrosion damage underneath protective coatings, by enabling the unexpected movement of ion-implanted tracers (such as Tc-99) to be detected and monitored.

Multifunctional paints can be formulated that enable the simultaneous detection of alpha, beta and gamma rays, with energy discrimination. While simple scintillation pigments provide a means of instantaneous detection, the use of thermal (thermo) luminescent materials can provide an integral measurement of radiation exposure, with a historical record of any radiation exposure that a particular coating may have been exposed to.

A vision has evolved where the surfaces of engineered structures are functionalized to enable them to serve as detectors of various environmental contaminants, including radioactive materials. Since the surfaces of most engineered structures are painted to protect them from corrosion, and to impart esthetic beauty, paints provide a viable means for imparting such functionality to surfaces. Ultimately, such paints could be used to allow relatively easy detection of widespread radioactive contamination on the surfaces of buildings and mass transit systems, thereby enabling such areas to be more readily decontaminated. Such coatings could also be used to form large-area temper-proof indicators, to reveal the presence of radioactive materials, and to ensure compliance with various regulatory requirements and other agreements. Such paints would be ideal for buildings and facilities involved in the processing and storage of nuclear materials, and could even be used to monitor contamination and dose on-board space craft and inside nuclear powered ships. Developmental work done thus far has demonstrated the viability of this concept. The concept of using sensing pigments can be easily extended to molded plastic parts. Additional work required for the full realization of the concepts potential is outlined.

By using such paints in tunnels and structures that might become contaminated with radioactive materials, decontamination efforts could be enhanced since the exact locations of contamination could be easily detected, using either a camera or more sensitive systems. The paints could be reset during the decontamination effort, so that progress could be monitored. There are many other attractive applications of such material. These paints are inexpensive, easy to deploy on massive surfaces, and easy to interrogate.

\section{FUTURE WORK}

Suitable applications should be identified in collaboration with potential end users.

The scintillation and thermo-luminescent type paints should both be optimized by: (1) matching the pigment to the radiation-detection application; (2) matching the refractive indices of the pigments and binders to minimize the luminescent photons scattered away from the detectors; (3) using only optimal pigment particles; and (4) preparing emulsions with the proper viscosity and drying characteristics for application with spray systems.

Multi-functional radiation-detection paints should be developed, with mixtures of pigments which could impart sensitivity to various types of radiation, including alpha, beta, gamma, and neutron. Pigments could also be modified to enable energy discrimination, which could be de-convoluted during interrogation.

Other nano-engineered materials and quantumdots, developed by the scientific comm could also be employed for the purpose of pigmentation.

A hand-held stand-off monitoring system, using a laser to heat a spot on the painted surface during interrogation, and a telescope and photomultiplier tube for simultaneously monitoring thermoluminescence should be developed.

\section{ACKNOWLEDGEMENTS}

The Defense Sciences Office (DSO) of the Defense Advanced Projects Agency (DARPA) sponsored this work. Lawrence Livermore National Laboratory (LLNL) performed this work under the auspices of the United States Department of Energy (DOE) by Lawrence Livermore National Laboratory (LLNL), through Contract No. W-7405-Eng-48.

Important recommendations from Steven Wax and Leo Christodoulou at DARPA DSO, Adrian Smith at DTI, and Milt Finger at LLNL are all gratefully acknowledged, and have enabled technical success.

The authors gratefully acknowledge the technical contributions of James L. Brunk, S. Daniel Day, and Phillip D. Hailey in the laboratory. Technical guidance and insight from Kathleen $\mathrm{H}$. Shingleton, LLNL Section Leader, Dosimetry, Hazards Control Department, and Patrick J. LaFrate, Health Physicist, LLNL Hazards Control Department, are also 
acknowledged for collaboration on the further development of the thermal luminescent paint concept.

\section{REFERENCES}

1. A. R. Foster, R. L. White, Jr., Scintillation Counters, Radiation Detection, Chapter 5, Basic Nuclear Engineering, Second Edition, Allyn \& Bacon, Boston, Massachusetts, 1973, Table 5.2, pp. 111-114.

2. L. Smart, E. Moore, An Introduction fo Simple Crystal Structures, Chapter 1, X-Ray Diffraction, Chapter 2, Defects and Non-Stoichiometry, Solid State Chemistry, $2^{\text {nd }}$ Edition, Stanley Thornes Publishers, Ltd., 1998, Ellenborough House, Wellington House, Wellington Street, Cheltenham, GL50 1YW, United Kingdom, pp. 35, 37, 44, 69, 81, 94, 161, 165, 186.

3. Sodium Iodide Scintillation Material, SaintGobain Ceramics and Plastics, Incorporated, Saint-Gobain Crystals, 12345 Kinsman Road, Newbury, Ohio 44065, Publication 3105(01-05), January 2005.

4. Cesium Iodide Scintillation Material, SaintGobain Ceramics and Plastics, Incorporated, Saint-Gobain Crystals, 12345 Kinsman Road, Newbury, Ohio 44065, Publication 3104(09-04), September 2004.

5. Calcium Fluoride Scintillation Material, SaintGobain Ceramics and Plastics, Incorporated, Saint-Gobain Crystals, 12345 Kinsman Road, Newbury, Ohio 44065, Publication 3103 (01-05), January 2005.

6. K. H. Shingleton, LLNL Hazards Control Department, Unpublished Meeting Notes, 2005.

7. Scintillation Products, Scintillation Materials and Assemblies, Saint-Gobain Ceramics and Plastics, Incorporated, Saint-Gobain Crystals, 12345 Kinsman Road, Newbury, Ohio 44065, Publication 3105(01-05), January 2005, pp. 1-11.

8. Z. W. Wicks, Jr., F. N. Jones, S. P. Pappas, Corrosion Protection by Coatings, Chapter 7, Latexes, Chapter 8, Binders Based on Isocyanates - Polyurethane, Chaper 10, Solventsw, Chapter 17, Color and Appearance, Chapter 18, Pigments, Chapter 19, Pigment Dispersion, Chapter 20, Organic Coatings, Science and Technology, $2^{\text {nd }}$ Edition, Wiley-Interscience, New York, New
York, 1999, pp. 125-138, 143-158, 180-207, 306334, 335-369, 370-384, 385-403.

9. J. C. Stewart, D. M. Hawcroft, Counting Radioisotopes, Chapter 3, Appendix 5, A Manual of Radiobiology, University of Washington Press, 1977, pp. 18-43, 191-192.

10. Physical Constants of Inorganic Compounds, Table of the Isotopes, Gamma Energies and Intensities of Radionuclides, $\mathrm{CRC}$ Handbook of Chemistry and Physics, $59^{\text {th }}$ Edition, R. C. Weast, Editor, M. J. Astle, Associate Editor, CRC Press, Incorporated, 2255 Palm Beach Lakes Boulevard, West Palm Beach, Florida 33409, 1977, pp. B105 , B-110, B-166, B-183, B-281, B-285, B-312B-344, B-352, B-363, B-364, B-381, B-410, B414 \& B-415.

11. E. Browne, R. B. Firestone, Table of Radioactive Isotopes, V. S. Shirley, Editor, John Wiley \& Sons.

12. Radionuclide Transformations, Energy and Intensity of Emissions, Report of a Task Group of Committee 2 of the International Commission on Radiological Protection on Data Used in ICRP Publication 30, The International Commission on Radiological Protection (ICRP), ICRP Publication 38, Pergamon Press, New York, NY, 1983, pp. 63, 501.

13. Radiological Health Handbook, Revised Edition, January 1970, United States Department of Health, Education and Welfare, Public Health Service, January, 1970, p. 113.

14. Linus Pauling, Nuclear Chemistry, Chapter 26, General Chemistry, Dover Publications, Incorporated, New York, 1970, pp. 831-863.

15. Diamond Elite Water Base (WB) Interior, Identification Numbers 200031, 200041, 200061, 200131, 200141, 200161, 200231, 200241, 200261, Material Safety Data Sheet, Rust-Oleum Corporation, 11 Hawthorne Parkway, Vernon Hills, Illinois 60061, April 18, 2003, 5 pages.

16. Varathane Interior, Identification Numbers 6031, 6041, 6051, 6061, 9131, 9151, 9161, 9031, 9041, 9051, 9061, 9141, Material Safety Data Sheet, Rust-Oleum Corporation, 11 Hawthorne Parkway, Vernon Hills, Illinois 60061, March 25, 2003, 5 pages. 